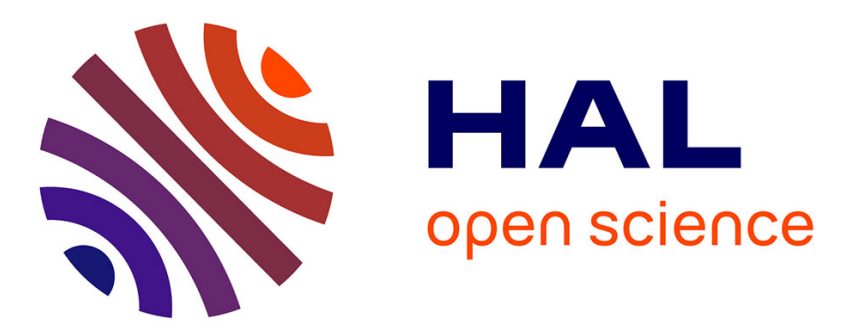

\title{
Explicit strategies for incompressible fluid-structure interaction problems: Nitsche type mortaring versus Robin-Robin coupling
}

\author{
Erik Burman, Miguel Angel Fernández
}

\section{- To cite this version:}

Erik Burman, Miguel Angel Fernández. Explicit strategies for incompressible fluid-structure interaction problems: Nitsche type mortaring versus Robin-Robin coupling. International Journal for Numerical Methods in Engineering, 2014, 97 (10), pp.739-758. 10.1002/nme.4607 . hal-00819948v2

\section{HAL Id: hal-00819948 \\ https://hal.inria.fr/hal-00819948v2}

Submitted on 29 Oct 2013

HAL is a multi-disciplinary open access archive for the deposit and dissemination of scientific research documents, whether they are published or not. The documents may come from teaching and research institutions in France or abroad, or from public or private research centers.
L'archive ouverte pluridisciplinaire HAL, est destinée au dépôt et à la diffusion de documents scientifiques de niveau recherche, publiés ou non, émanant des établissements d'enseignement et de recherche français ou étrangers, des laboratoires publics ou privés. 
Explicit strategies for incompressible fluid-structure interaction problems: Nitsche type mortaring versus Robin-Robin coupling

Erik Burman, Miguel A. Fernández

RESEARCH REPORT

$\mathrm{N}^{\circ} 8296$

May 2013 



\title{
Inĩá
}

\section{Explicit strategies for incompressible fluid-structure interaction problems: Nitsche type mortaring versus Robin-Robin coupling}

\author{
Erik Burman*, Miguel A. Fernández ${ }^{\dagger \ddagger}$ \\ Project-Team Reo \\ Research Report n8296 - May 2013 - 26 pages
}

\begin{abstract}
We discuss explicit coupling schemes for fluid-structure interaction problems where the added mass effect is important. In this paper we show the close relation between coupling schemes using Nitsche's method and a Robin-Robin type coupling. In the latter case the method may be implemented either using boundary integrals of the stresses or the more conventional discrete lifting operators. Recalling the explicit method proposed in Comput. Methods Appl. Mech. Engrg. 198(5-8):766-784, 2009 we make the observation that this scheme is stable under a hyperbolic type CFL condition, but that optimal accuracy imposes a parabolic type CFL conditions due to the splitting error. Two strategies to enhance the accuracy of the coupling scheme under the hyperbolic CFL-condition are suggested, one using extrapolation and defect-correction and one using a penalty-free non-symmetric Nitsche method. Finally we illustrate the performance of the proposed schemes on some numerical examples in two and three space dimensions.
\end{abstract}

Key-words: fluid-structure interaction, incompressible fluid, time-discretization, loosely coupled scheme, Robin-Robin scheme, finite element method, Nitsche's method.

The first author received funding for this research from EPSRC (award number EP/J002313/1). The second author was financially supported by the French National Research Agency (ANR) through the EXIFSI project (ANR-12-JS01-0004).

* Department of Mathematics, University College London, London, UK-WC1E 6BT, United Kingdom

$\dagger$ Inria, REO project-team, Rocquencourt - B.P. 105, F-78153 Le Chesnay cedex, France

‡ UPMC Université Paris VI, REO project-team, UMR 7958 LJLL, F-75005 Paris, France

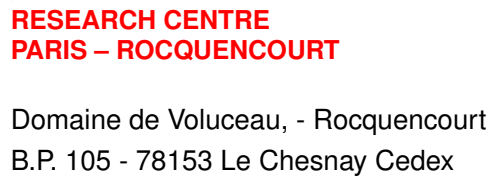




\section{Schémas explicites pour l'interaction d'un fluide incompressible avec une structure élastique: couplage à la Nitsche $v s$. couplage Robin-Robin}

Résumé : Nous étudions quelques schémas de couplage explicites pour des problèmes d'interaction fluide-structure avec un effet de masse-ajoutée important. Pour la méthode explicite proposée dans Comput. Methods Appl. Mech. Engrg. 198(5-8):766-784, 2009 nous observons que ce schéma est stable sous une CFL hyperbolique, mais que la précision optimale impose un condition CFL de type parabolique due à l'erreur de découplage. Dans cet article, nous montrons une relation intrinsèque entre schémas de couplage utilisant la méthode de Nitsche et un couplage de type Robin-Robin. Dans ce dernier cas, la méthode peut être mise en œuvre en intégrant les efforts sur l'interface ou à l'aide d'opérateurs de relèvement discrets plus classiques. Deux stratégies pour améliorer la précision du découplage sous une condition CFL hyperbolique sont proposées : une via extrapolation et correction et l'autre via une méthode Nitsche nonsymétrique sans pénalisation. Enfin, nous illustrons les performances des schémas proposés sur quelques exemples numériques en deux et trois dimensions d'espace.

Mots-clés : interaction fluide-structure, fluide incompressible, discrétisation en temps, schéma faiblement couplé, schéma Robin-Robin, méthode des éléments, méthode de Nitsche. 


\section{Introduction}

Incompressible fluid-structure interaction (IFSI), that is, the coupling of an elastic structure with an incompressible fluid, appears in a variety of engineering fields, from the aeroelasticity of bridge decks and parachutes, to naval hydrodynamics and the biomechanics of blood flow. Over the last decade, the development of efficient numerical methods for IFSI has been a very active field of research and the subject of numerous works (see, e.g., [13, 26] for recent reviews).

This is due, in particular, to the fact that the coupling is known to be very stiff. So called explicit (or loosely coupled, see [33, 34, 12]) schemes, where time advancement is obtained by first taking one time-step in the fluid and then one in the structure, using the data from the fluid solve, are known to be unstable for standard Dirichlet-Neumann coupling strategies where the amount of added-mass in the system is strong (see, e.g., [7, 21]). In view of this, much research effort has gone into the design of robust solvers for the solution of the more computationally onerous implicit and semi-implicit coupling paradigms (see, e.g., [28, 23, 25, 16, 10, 9, 15, 36, 3, $27,30,2,24,22,8,31,29])$.

In order to pass to a fully explicit coupling scheme, the interface conditions must be treated in a different fashion. In [6] we proposed a loosely coupled scheme for the solution of fluid structure interaction problems using a Nitsche type weak coupling together with an interface pressure stabilization in time. It was shown that the combination of these two terms leads to an unconditionally stable scheme independent of the added-mass effect in the system. In the case of the coupling with a thin-walled structure, alternative stable explicit coupling schemes are the Robin-Neumann methods proposed in $[14,18]$ which yield optimal first-order accuracy in time. A preliminary extension of these methods to the case of the coupling with thick solid models has been reported in [17], but in this case the splitting does not preserve first-order accuracy.

Similarly, the main drawback of the Nitsche based staggered scheme proposed in [6] is the introduction of a splitting error that puts constraints on how the time-step must be chosen compared to the space-step to obtain first-order accuracy. The conditions required for accuracy are often stronger than those required for stability in these cases. The stability condition is related to the hyperbolic nature of the elasticity problem whereas the accuracy relies on the resolution of the coupling through the viscous term of the parabolic system.

Typically a formal error analysis for piecewise linear finite elements and a first order timediscretization method results in an error of order

$$
\underbrace{(h \tau)^{\frac{1}{2}}}_{\text {pressure stabilization }}+\underbrace{\frac{\tau}{h}}_{\text {Nitsche coupling }}+\underbrace{h^{s}+\tau}_{\text {order of scheme }},
$$

with $s=1$ for error estimates on the stresses and $s=2$ for $L^{2}$-error estimates on structure displacements or fluid velocities. Clearly to obtain $O(h)$ accuracy, we need to choose $\tau=O\left(h^{2}\right)$, corresponding to a parabolic constraint on the time-step. Using a defect correction strategy, with $k$ correction steps (see [6, Section 5.4]), this error can be improved to

$$
\underbrace{(h \tau)^{\frac{k+1}{2}}}_{\text {pressure stabilization }}+\underbrace{\left(\frac{\tau}{h}\right)^{k+1}}_{\text {Nitsche coupling }}+\underbrace{h^{s}+\tau}_{\text {order of scheme }}
$$

and it follows that the choice $\tau=O\left(h^{2}\right)$ leads to optimal convergence for the $L^{2}$-error after one correction step. One advantage of the explicit coupling scheme based on Nitsche's method is that it does not use any dissipation of the time discretization for stability. It is therefore in principle possible to improve the time accuracy above to $O\left(\tau^{2}\right)$, such an improvement is of little use if we can not improve on the splitting error of order $O(\tau / h)$. 
In this paper we wish to adress the question of how to improve the accuracy of the explicit coupling scheme at a modest cost and study modifications that make the scheme easier to implement in standard finite element codes. In particular we will study:

- The close relationship of the Nitsche based coupling method with Robin type coupling methods and with the standard variational framework, where stresses are computed using lifting operators and not using face-wise integrals. This results in loosely coupled algorithms, containing fewer non-standard features than the original Nitsche-coupling scheme;

- The possibility of enhancing accuracy using extrapolation and defect correction;

- The possibility to reduce stabilization, in order to diminish the consistency errors. We suggest one Robin based scheme without pressure stabilization term and a non-symmetric Nitsche type scheme without penalty on the velocity.

There appears to be a wide range of possibilities to explore in the framework of explicit coupling schemes and the aim of this paper is to show some variants, point to some of the difficulties associated with explicit coupling and discuss some possible remedies. An outline of the paper is as follows. First we introduce a linear model problem, then in Section 3 we recall the Nitsche based explicit coupling procedure of [6] and show how it can be interpreted as a Robin type coupling using face-wise or residual based coupling. In Section 4 we discuss some strategies for enhancing accuracy. We illustrate the discussion with some numerical investigations in Section 5. Finally, Section 6 draws the conclusions and a few lines for further investigation.

\section{A linear model problem}

We consider a low Reynolds regime and assume that the interface undergoes infinitesimal displacements. The fluid is described by the Stokes equations, in a fixed domain $\Omega_{\mathrm{f}} \subset \mathbb{R}^{d}, d=2,3$,

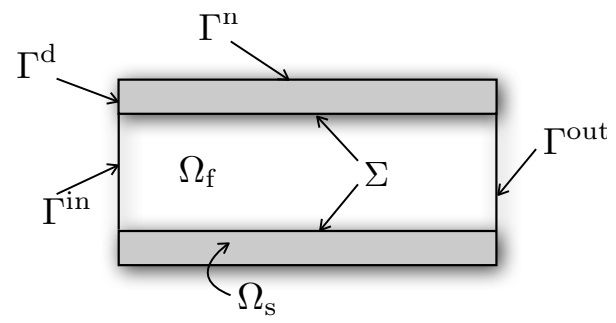

Figure 1: Geometrical description

and the structure by the classical linear elasticity equations, in the solid domain $\Omega_{\mathrm{s}} \subset \mathbb{R}^{d}$. We denote by $\Sigma \stackrel{\text { def }}{=} \partial \Omega_{\mathrm{s}} \cap \partial \Omega_{\mathrm{f}}$ the fluid-structure interface and $\partial \Omega_{\mathrm{f}} \stackrel{\text { def }}{=} \Gamma^{\text {in }} \cup \Gamma^{\text {out }} \cup \Sigma, \partial \Omega_{\mathrm{s}} \stackrel{\text { def }}{=} \Gamma^{\mathrm{d}} \cup \Gamma^{\mathrm{n}} \cup \Sigma$, are given partitions of the fluid and solid boundaries, respectively (see Figure 1). Our simplified coupled problem reads as follows: Find the fluid velocity $\boldsymbol{u}: \Omega_{\mathrm{f}} \times \mathbb{R}^{+} \rightarrow \mathbb{R}^{d}$, the fluid pressure $p: \Omega_{\mathrm{f}} \times \mathbb{R}^{+} \rightarrow \mathbb{R}$, the structure displacement $\boldsymbol{d}: \Omega_{\mathrm{s}} \times \mathbb{R}^{+} \rightarrow \mathbb{R}^{d}$ and the structure velocity $\dot{\boldsymbol{d}}: \Omega_{\mathrm{s}} \times \mathbb{R}^{+} \rightarrow \mathbb{R}^{d}$ such that

$$
\left\{\begin{array}{rcc}
\rho_{\mathrm{f}} \partial_{t} \boldsymbol{u}-\operatorname{div} \boldsymbol{\sigma}(\boldsymbol{u}, p)=\mathbf{0} & \text { in } \quad \Omega_{\mathrm{f}}, \\
\operatorname{div} \boldsymbol{u}=0 & \text { in } & \Omega_{\mathrm{f}} \\
\boldsymbol{u}=\boldsymbol{u}_{\mathrm{in}} & \text { on } & \Gamma^{\text {in }} \\
\boldsymbol{\sigma}(\boldsymbol{u}, p) \boldsymbol{n}=-p_{\text {out }} \boldsymbol{n} & \text { on } & \Gamma^{\text {out }}
\end{array}\right.
$$




$$
\left\{\begin{array}{rcc}
\rho_{\mathrm{s}} \partial_{t} \dot{\boldsymbol{d}}-\operatorname{div} \boldsymbol{\sigma}(\boldsymbol{d})+\beta \boldsymbol{d}=\mathbf{0} & \text { in } & \Omega_{\mathrm{s}}, \\
\dot{\boldsymbol{d}}=\partial_{t} \boldsymbol{d} & \text { in } & \Omega_{\mathrm{s}} \\
\boldsymbol{d}=\mathbf{0} & \text { on } & \Gamma^{\mathrm{d}} \\
\boldsymbol{\sigma}(\boldsymbol{d}) \boldsymbol{n}_{\mathrm{s}}=\mathbf{0} & \text { on } & \Gamma^{\mathrm{n}}
\end{array}\right.
$$

satisfying the interface coupling conditions

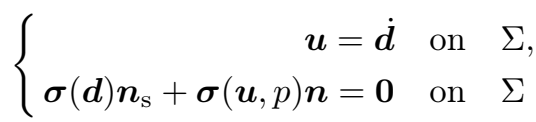

and the initial conditions $\boldsymbol{u}(0)=\boldsymbol{u}_{0}, \boldsymbol{d}(0)=\boldsymbol{d}_{0}$ and $\dot{\boldsymbol{d}}(0)=\boldsymbol{v}_{0}$. Here, $\rho_{\mathrm{f}}$ and $\rho_{\mathrm{s}}$ stand for the fluid and solid densities, $\boldsymbol{\sigma}(\boldsymbol{u}, p) \stackrel{\text { def }}{=}-p \boldsymbol{I}+2 \mu \boldsymbol{\epsilon}(\boldsymbol{u})$ for the fluid stress tensor, $\mu$ for the fluid dynamic viscosity, $\boldsymbol{\epsilon}(\boldsymbol{u}) \stackrel{\text { def }}{=} \frac{1}{2}\left(\boldsymbol{\nabla} \boldsymbol{u}+\boldsymbol{\nabla} \boldsymbol{u}^{\mathrm{T}}\right)$ for the fluid strain rate tensor, $\boldsymbol{u}_{\text {in }}$ for a given velocity profile, $p_{\text {out }}$ for a given outlet pressure and $\boldsymbol{n}, \boldsymbol{n}_{\mathrm{s}}$ for the exterior unit normal vectors to the boundaries of $\Omega_{\mathrm{f}}$ and $\Omega_{\mathrm{s}}$, respectively. The solid stress tensor is given by $\boldsymbol{\sigma}(\boldsymbol{d}) \stackrel{\text { def }}{=} 2 L_{1} \boldsymbol{\epsilon}(\boldsymbol{d})+\left(L_{2} \operatorname{div} \boldsymbol{d}\right) \boldsymbol{I}$ with $L_{1}, L_{2}>0$ the Lamé constants. The zeroth-order term in $(2)_{1}$, with $\beta \geq 0$, allows to incorporate the transversal membrane effects that appear in axisymmetric formulations.

\section{Time-discretization: explicit coupling procedures}

We first recall the original loosely coupled algorithm introduced in [6] for the solution of (1)-(3) and then show how a reduction of this scheme leads to a Robin-type coupling scheme, using residual based stress computation. We also prove that the latter variant preserves the (addedmass free) stability properties of the original scheme.

\subsection{The Nitsche based explicit coupling procedure}

Let $\boldsymbol{V}_{\mathrm{f}, h} \times Q_{h}$ denote a conforming, inf-sup stable, finite element approximation of $\left[H^{1}\left(\Omega_{\mathrm{f}}\right)\right]^{d} \times$ $L^{2}\left(\Omega_{\mathrm{f}}\right)$, and $\boldsymbol{V}_{\mathrm{s}, h}$ a conforming finite element approximation of $\left[H_{\Gamma^{d}}^{1}\left(\Omega_{\mathrm{s}}\right)\right]^{d}$. We also introduce the space $\boldsymbol{V}_{\mathrm{f}, \Gamma^{\text {in }}, h} \stackrel{\text { def }}{=} \boldsymbol{V}_{\mathrm{f}, h} \cap\left[H_{\Gamma^{\text {in }}}^{1}\left(\Omega_{\mathrm{f}}\right)\right]^{d}$. We consider a first order backward difference discretization in the fluid and the structure, so that the fully discrete fluid and solid bilinear forms at time level $n, 1 \leq n \leq N$, are given by:

$$
\begin{gathered}
A_{\mathrm{f}, \tau}\left(\left(\boldsymbol{u}_{h}^{n}, p_{h}^{n}\right),\left(\boldsymbol{v}_{h}, q_{h}\right)\right) \stackrel{\text { def }}{=} \rho_{\mathrm{f}} \int_{\Omega_{\mathrm{f}}} \partial_{\tau} \boldsymbol{u}_{h}^{n} \cdot \boldsymbol{v}_{h}+\int_{\Omega_{\mathrm{f}}} \boldsymbol{\sigma}\left(\boldsymbol{u}_{h}^{n}, p_{h}^{n}\right): \boldsymbol{\epsilon}\left(\boldsymbol{v}_{h}\right)+\int_{\Omega_{\mathrm{f}}} \operatorname{div} \boldsymbol{u}_{h}^{n} q_{h} \\
A_{\mathrm{s}, \tau}\left(\left(\boldsymbol{d}_{h}^{n}, \dot{\boldsymbol{d}}_{h}^{n}\right),\left(\boldsymbol{w}_{h}, \boldsymbol{z}_{h}\right)\right) \stackrel{\text { def }}{=} \rho_{\mathrm{s}} \int_{\Omega_{\mathrm{s}}} \partial_{\tau} \dot{\boldsymbol{d}}_{h}^{n} \cdot \boldsymbol{w}_{h}+a_{\mathrm{s}}\left(\boldsymbol{d}_{h}^{n}, \boldsymbol{w}_{h}\right)+\int_{\Omega_{\mathrm{s}}}\left(\dot{\boldsymbol{d}}_{h}^{n}-\partial_{\tau} \boldsymbol{d}_{h}^{n}\right) \cdot \boldsymbol{z}_{h} .
\end{gathered}
$$

Here $\partial_{\tau}$ denotes the first order backward difference operator, $\partial_{\tau} x^{n} \stackrel{\text { def }}{=}\left(x^{n}-x^{n-1}\right) / \tau, \tau>0$ the time-step length and

$$
a_{\mathrm{s}}(\boldsymbol{d}, \boldsymbol{w}) \stackrel{\text { def }}{=} \int_{\Omega_{\mathrm{s}}} \boldsymbol{\sigma}(\boldsymbol{d}): \boldsymbol{\epsilon}(\boldsymbol{w})
$$

The original stabilized explicit coupling scheme, reported in [6], is presented in Algorithm 1. 
Algorithm 1 Nitsche based stabilized explicit coupling (from [6]).

1. Solid sub-problem: find $\boldsymbol{d}_{h}^{n}, \dot{\boldsymbol{d}}_{h}^{n} \in \boldsymbol{V}_{\mathrm{s}, h}$ such that

$$
A_{\mathrm{s}, \tau}\left(\left(\boldsymbol{d}_{h}^{n}, \dot{\boldsymbol{d}}_{h}^{n}\right),\left(\boldsymbol{w}_{h}, \boldsymbol{z}_{h}\right)\right)+\gamma \frac{\mu}{h} \int_{\Sigma} \dot{\boldsymbol{d}}_{h}^{n} \cdot \boldsymbol{w}_{h}=\gamma \frac{\mu}{h} \int_{\Sigma} \boldsymbol{u}_{h}^{n-1} \cdot \boldsymbol{w}_{h}-\int_{\Sigma} \boldsymbol{\sigma}\left(\boldsymbol{u}_{h}^{n-1}, p_{h}^{n-1}\right) \boldsymbol{n} \cdot \boldsymbol{w}_{h}
$$

for all $\boldsymbol{w}_{h}, \boldsymbol{z}_{h} \in \boldsymbol{V}_{\mathrm{s}, h}$.

2. Fluid sub-problem: find $\left(\boldsymbol{u}_{h}^{n}, p_{h}^{n}\right) \in \boldsymbol{V}_{\mathrm{f}, h} \times Q_{h}$, with $\left.\boldsymbol{u}_{h}^{n}\right|_{\Gamma^{\text {in }}}=\boldsymbol{u}_{\text {in }}^{n}$, such that

$$
\begin{array}{r}
A_{\mathrm{f}, \tau}\left(\left(\boldsymbol{u}_{h}^{n}, p_{h}^{n}\right),\left(\boldsymbol{v}_{h}, q_{h}\right)\right)+\gamma \frac{\mu}{h} \int_{\Sigma} \boldsymbol{u}_{h}^{n} \cdot \boldsymbol{v}_{h}-\int_{\Sigma} \boldsymbol{u}_{h}^{n} \cdot \boldsymbol{\sigma}\left(\boldsymbol{v}_{h},-q_{h}\right) \boldsymbol{n}+\frac{\gamma_{0} h}{\gamma \mu} \int_{\Sigma} p_{h}^{n} q_{h} \\
=\gamma \frac{\mu}{h} \int_{\Sigma} \dot{\boldsymbol{d}}_{h}^{n} \cdot \boldsymbol{v}_{h}-\int_{\Sigma} \dot{\boldsymbol{d}}_{h}^{n} \cdot \boldsymbol{\sigma}\left(\boldsymbol{v}_{h},-q_{h}\right) \boldsymbol{n}+\frac{\gamma_{0} h}{\gamma \mu} \int_{\Sigma} p_{h}^{n-1} q_{h}+\int_{\Sigma} \boldsymbol{\sigma}\left(\boldsymbol{u}_{h}^{n-1}, p_{h}^{n-1}\right) \boldsymbol{n} \cdot \boldsymbol{v}_{h} \\
-\int_{\Gamma_{\text {out }}} p_{\text {out }}\left(t_{n}\right) \boldsymbol{v}_{h} \cdot \boldsymbol{n}
\end{array}
$$

for all $\left(\boldsymbol{v}_{h}, q_{h}\right) \in \boldsymbol{V}_{\mathrm{f}, \Gamma^{\mathrm{in}}, h} \times Q_{h}$.

The stability properties of Algorithm 1 are stated in the following result, from [6, Theorem 9], where

$$
E^{n} \stackrel{\text { def }}{=} \frac{\rho_{\mathrm{f}}}{2}\left\|\boldsymbol{u}_{h}^{n}\right\|_{0, \Omega_{\mathrm{f}}}^{2}+\frac{\rho_{\mathrm{s}}}{2}\left\|\dot{\boldsymbol{d}}_{h}^{n}\right\|_{0, \Omega_{\mathrm{s}}}^{2}+\frac{1}{2} a_{\mathrm{s}}\left(\boldsymbol{d}_{h}^{n}, \boldsymbol{d}_{h}^{n}\right)
$$

denotes the total discrete energy of the system at time level $n$ and the symbols $\lesssim$ and $\gtrsim$ indicate inequalities up to a multiplicative constant (independent of the physical and discretization parameters).

Theorem 1 Assume that the fluid-structure system is isolated, i.e., $p_{\text {out }}=0$ and $\boldsymbol{u}_{\text {in }}=\mathbf{0}$. Let $\left\{\left(\boldsymbol{u}_{h}^{n}, p_{h}^{n}, \boldsymbol{d}_{h}^{n}, \dot{\boldsymbol{d}}_{h}^{n}\right)\right\}_{1 \leq n \leq N}$ be given by Algorithm 1. Then, under the following conditions

$$
\gamma \gtrsim C_{\mathrm{ti}}, \quad \gamma \tau \lesssim h, \quad \gamma_{0} \gtrsim 1,
$$

where $C_{\mathrm{ti}}$ is a trace/inverse estimate constant, there holds

$$
E^{n} \lesssim E^{0}+\mu\left\|\boldsymbol{u}_{h}^{0}\right\|_{0, \Sigma}^{2}+\mu\left\|\boldsymbol{\epsilon}\left(\boldsymbol{u}_{h}^{0}\right)\right\|_{0, \Omega_{\mathrm{f}}}^{2}+\frac{\gamma_{0} h}{\gamma \mu} \tau\left\|p_{h}^{0}\right\|_{0, \Sigma}^{2}
$$

for $1 \leq n \leq N$.

The main source of splitting error in Algorithm 1 comes from the penalty term

$$
\gamma \frac{\mu}{h} \int_{\Sigma} \boldsymbol{u}_{h}^{n-1} \cdot \boldsymbol{w}_{h}
$$

in the right hand side of (4). Indeed this is the term that results in an error contribution of the form $\mathcal{O}(\tau / h)$ and hence the need for the time step to be chosen small enough compared to the space step. 


\subsection{Stabilized Robin-Robin explicit coupling}

Nitsche's method consists of three boundary terms, one term that guarantees consistency of the formulation, one that penalizes the jump in the velocities between the fluid and the structure and finally one term that is added in an ad hoc fashion to preserve the symmetry of the stress tensor on the discrete level. The energy stability result of Theorem 1 still remains valid if in (5) we neglect the viscous contribution of the "symmetrizing" term

$$
-\int_{\Sigma}\left(\boldsymbol{u}_{h}^{n}-\dot{\boldsymbol{d}}_{h}^{n}\right) \cdot \boldsymbol{\sigma}\left(\boldsymbol{v}_{h},-q_{h}\right) \boldsymbol{n},
$$

resulting in the term

$$
-\int_{\Sigma} q_{h}\left(\boldsymbol{u}_{h}^{n}-\dot{\boldsymbol{d}}_{h}^{n}\right) \cdot \boldsymbol{n}
$$

which can be interpreted as a weak imposition of the non-penetration interface constraint

$$
\left(\boldsymbol{u}_{h}^{n}-\dot{\boldsymbol{d}}_{h}^{n}\right) \cdot \boldsymbol{n}=0 \quad \text { on } \quad \Sigma .
$$

This yields the solution procedure reported in Algorithm 2.

Algorithm 2 Stabilized Robin-Robin explicit coupling.

1. Solid sub-problem: find $\boldsymbol{d}_{h}^{n}, \dot{\boldsymbol{d}}_{h}^{n} \in \boldsymbol{V}_{\mathrm{s}, h}$ such that

$$
A_{\mathrm{s}, \tau}\left(\left(\boldsymbol{d}_{h}^{n}, \dot{\boldsymbol{d}}_{h}^{n}\right),\left(\boldsymbol{w}_{h}, \boldsymbol{z}_{h}\right)\right)+\gamma \frac{\mu}{h} \int_{\Sigma} \dot{\boldsymbol{d}}_{h}^{n} \cdot \boldsymbol{w}_{h}=\gamma \frac{\mu}{h} \int_{\Sigma} \boldsymbol{u}_{h}^{n-1} \cdot \boldsymbol{w}_{h}-\int_{\Sigma} \boldsymbol{\sigma}\left(\boldsymbol{u}_{h}^{n-1}, p_{h}^{n-1}\right) \boldsymbol{n} \cdot \boldsymbol{w}_{h}
$$

for all $\boldsymbol{w}_{h}, \boldsymbol{z}_{h} \in \boldsymbol{V}_{\mathrm{s}, h}$.

2. Fluid sub-problem: find $\left(\boldsymbol{u}_{h}^{n}, p_{h}^{n}\right) \in \boldsymbol{V}_{\mathrm{f}, h} \times Q_{h}$, with $\left.\boldsymbol{u}_{h}^{n}\right|_{\Gamma^{\text {in }}}=\boldsymbol{u}_{\text {in }}^{n}$, such that

$$
\begin{aligned}
& A_{\mathrm{f}, \tau}\left(\left(\boldsymbol{u}_{h}^{n}, p_{h}^{n}\right),\left(\boldsymbol{v}_{h}, q_{h}\right)\right)+\gamma \frac{\mu}{h} \int_{\Sigma} \boldsymbol{u}_{h}^{n} \cdot \boldsymbol{v}_{h}-\int_{\Sigma} \boldsymbol{u}_{h}^{n} \cdot \boldsymbol{n} q_{h}+\frac{\gamma_{0} h}{\gamma \mu} \int_{\Sigma} p_{h}^{n} q_{h} \\
& =\gamma \frac{\mu}{h} \int_{\Sigma} \dot{\boldsymbol{d}}_{h}^{n} \cdot \boldsymbol{v}_{h}-\int_{\Sigma} \dot{\boldsymbol{d}}_{h}^{n} \cdot \boldsymbol{n} q_{h}+\frac{\gamma_{0} h}{\gamma \mu} \int_{\Sigma} p_{h}^{n-1} q_{h}+\int_{\Sigma} \boldsymbol{\sigma}\left(\boldsymbol{u}_{h}^{n-1}, p_{h}^{n-1}\right) \boldsymbol{n} \cdot \boldsymbol{v}_{h} \\
& -\int_{\Gamma \text { out }} p_{\text {out }}^{n} \boldsymbol{v}_{h} \cdot \boldsymbol{n}
\end{aligned}
$$

for all $\left(\boldsymbol{v}_{h}, q_{h}\right) \in \boldsymbol{V}_{\mathrm{f}, \Gamma^{\text {in }}, h} \times Q_{h}$.

A salient feature of this reduced variant of Algorithm 1 is that it can be viewed as a space discrete counterpart of the following Robin-Robin based explicit treatment of (3):

$$
\left.\begin{array}{rl}
\boldsymbol{\sigma}\left(\boldsymbol{d}^{n}\right) \boldsymbol{n}_{\mathrm{s}}+\frac{\gamma \mu}{h} \dot{\boldsymbol{d}}^{n} & =\frac{\gamma \mu}{h} \boldsymbol{u}^{n-1}-\boldsymbol{\sigma}\left(\boldsymbol{u}^{n-1}, p^{n-1}\right) \boldsymbol{n} \\
\boldsymbol{\sigma}\left(\boldsymbol{u}^{n}, p^{n}\right) \boldsymbol{n}+\frac{\gamma \mu}{h} \boldsymbol{u}^{n} & =\frac{\gamma \mu}{h} \dot{\boldsymbol{d}}_{h}^{n}+\boldsymbol{\sigma}\left(\boldsymbol{u}^{n-1}, p^{n-1}\right) \boldsymbol{n}
\end{array}\right\} \quad \text { on } \quad \Sigma,
$$

complemented with the following weakly-consistent interface compressibility treatment of the continuity equation $(1)_{2}$ :

$$
\operatorname{div} \boldsymbol{u}^{n}+\frac{\gamma_{0} h \tau}{\gamma \mu} \partial_{\tau} p^{n} \delta_{\Sigma}=\left(\boldsymbol{u}^{n}-\dot{\boldsymbol{d}}^{n}\right) \cdot \boldsymbol{n} \delta_{\Sigma}
$$

$\mathrm{RR} \mathrm{n}^{\circ} 8296$ 
where $\delta_{\Sigma}$ stands for the Dirac measure on the surface $\Sigma$. In other words, the constraint $\operatorname{div} \boldsymbol{u}^{n}=0$ is imposed in the volume $\Omega_{\mathrm{f}}$ and the constraint

$$
\boldsymbol{u}^{n} \cdot \boldsymbol{n}=\dot{\boldsymbol{d}}^{n} \cdot \boldsymbol{n}+\frac{\gamma_{0} h \tau}{\gamma \mu} \partial_{\tau} p^{n}
$$

is enforced on $\Sigma$.

Remark 1 The modified continuity equation (7) introduces an artificial compressibility in the spirit of [11], but with two important differences. First, it is restricted to the interface and, second, it is weakly consistent with the original continuous problem.

This novel interpretation of the Nitsche based coupling of Algorithm 1, raises two questions:

1. Can stabilized explicit coupling be appropriately implemented within a non-Nitsche's framework, that is, by using a variationally consistent formulation without face-wise interface stress computations?

2. What is the minimal stabilization required to obtain a stable explicit coupling scheme?

As we shall see below the first question has an affirmative answer based on the stabilized explicit coupling paradigm defined by (6)-(7). For the last question, in Section 3.3 we will discuss a modified Robin-Robin coupling scheme where numerical experiences indicate that the pressure stabilization term can be omitted entirely.

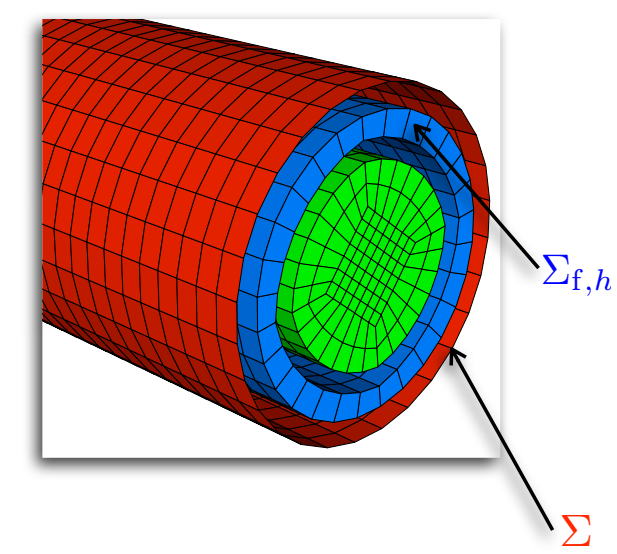

Figure 2: The fluid layer of elements, $\Sigma_{\mathrm{f}, h}$, adjacent to the fluid-solid interface $\Sigma$.

For the sake of simplicity, we assume that the fluid and the solid discrete spaces $\boldsymbol{V}_{\mathrm{f}, h}, \boldsymbol{V}_{\mathrm{s}, h}$ match at the interface $\Sigma$, that is, they share the same interface trace space $\boldsymbol{V}_{h}(\Sigma) \stackrel{\text { def }}{=} \operatorname{Tr}_{\Sigma}\left(\boldsymbol{V}_{\mathrm{s}, h}\right)=$ $\operatorname{Tr}_{\Sigma}\left(\boldsymbol{V}_{\mathrm{f}, h}\right)$. Let then

$$
\mathcal{L}_{h}: \boldsymbol{V}_{h}(\Sigma) \longrightarrow \boldsymbol{V}_{\mathrm{f}, h} \cap \boldsymbol{H}_{\partial \Omega_{\mathrm{f}}-\Sigma}^{1}\left(\Omega_{\mathrm{f}}\right)
$$

be the usual fluid-sided discrete lifting operator, such that

$$
\left.\mathcal{L}_{h} \boldsymbol{\xi}_{h}\right|_{\Sigma}=\boldsymbol{\xi}_{h},\left.\quad \mathcal{L}_{h} \boldsymbol{\xi}_{h}\right|_{\Omega_{\mathrm{f}}-\Sigma_{\mathrm{f}, h}}=\mathbf{0},
$$

where $\Sigma_{\mathrm{f}, h} \subset \Omega_{\mathrm{f}}$ stands for the fluid layer of elements adjacent to the fluid-solid interface $\Sigma$, see Figure 2. 
Algorithm 3 Stabilized Robin-Robin explicit coupling (without face-wise stress integration).

1. Solid sub-problem: find $\boldsymbol{d}_{h}^{n}, \dot{\boldsymbol{d}}_{h}^{n} \in \boldsymbol{V}_{\mathrm{s}, h}$ such that

$$
\begin{aligned}
A_{\mathrm{s}, \tau}\left(\left(\boldsymbol{d}_{h}^{n}, \dot{\boldsymbol{d}}_{h}^{n}\right),\left(\boldsymbol{w}_{h}, \boldsymbol{z}_{h}\right)\right)+\gamma \frac{\mu}{h} \int_{\Sigma} \dot{\boldsymbol{d}}_{h}^{n} \cdot \boldsymbol{w}_{h} \\
\quad=\gamma \frac{\mu}{h} \int_{\Sigma} \boldsymbol{u}_{h}^{n-1} \cdot \boldsymbol{w}_{h}-A_{\mathrm{f}, \tau}\left(\left(\boldsymbol{u}_{h}^{n-1}, p_{h}^{n-1}\right),\left(\mathcal{L}_{h} \boldsymbol{w}_{h}, 0\right)\right)
\end{aligned}
$$

for all $\boldsymbol{w}_{h}, \boldsymbol{z}_{h} \in \boldsymbol{V}_{\mathrm{s}, h}$.

2. Fluid sub-problem: find $\left(\boldsymbol{u}_{h}^{n}, p_{h}^{n}\right) \in \boldsymbol{V}_{\mathrm{f}, h} \times Q_{h}$, with $\left.\boldsymbol{u}_{h}^{n}\right|_{\Gamma^{\text {in }}}=\boldsymbol{u}_{\text {in }}\left(t_{n}\right)$, such that

$$
\begin{aligned}
A_{\mathrm{f}, \tau}\left(\left(\boldsymbol{u}_{h}^{n}, p_{h}^{n}\right),\left(\boldsymbol{v}_{h}, q_{h}\right)\right)+\gamma \frac{\mu}{h} \int_{\Sigma} \boldsymbol{u}_{h}^{n} \cdot \boldsymbol{v}_{h}-\int_{\Sigma_{\mathrm{f}, h}} \operatorname{div}\left(\mathcal{L}_{h} \boldsymbol{u}_{h}^{n}\right) q_{h}+\frac{\gamma_{0}}{\gamma \mu} \int_{\Sigma_{\mathrm{f}, h}} p_{h}^{n} q_{h} \\
=\gamma \frac{\mu}{h} \int_{\Sigma} \dot{\boldsymbol{d}}_{h}^{n} \cdot \boldsymbol{v}_{h}+\frac{\gamma_{0}}{\gamma \mu} \int_{\Sigma_{\mathrm{f}, h}} p_{h}^{n-1} q_{h}-\int_{\Sigma_{\mathrm{f}, h}} \operatorname{div}\left(\mathcal{L}_{h} \dot{\boldsymbol{d}}_{h}^{n}\right) q_{h} \\
+A_{\mathrm{f}, \tau}\left(\left(\boldsymbol{u}_{h}^{n-1}, p_{h}^{n-1}\right),\left(\mathcal{L}_{h} \boldsymbol{v}_{h}, 0\right)\right)-\int_{\Gamma_{\text {out }}} p_{\text {out }}^{n} \boldsymbol{v}_{h} \cdot \boldsymbol{n}
\end{aligned}
$$

for all $\left(\boldsymbol{v}_{h}, q_{h}\right) \in \boldsymbol{V}_{\mathrm{f}, \Gamma^{\text {in }}, h} \times Q_{h}$.

We now propose to implement the splitting (6)-(7) in a standard variational framework (i.e., without face-wise stress computations) thanks to the lifting operator $\mathcal{L}_{h}$. The resulting explicit coupling scheme is reported in Algorithm 3.

Some observations are now in order. The solid sub-step (8) is nothing but the discrete counterpart of $(6)_{1}$. As usual, the interface integral

$$
\int_{\Sigma} \boldsymbol{\sigma}\left(\boldsymbol{u}_{h}^{n-1}, p_{h}^{n-1}\right) \boldsymbol{n} \cdot \boldsymbol{w}_{h}
$$

is here replaced by the variationally consistent residual approximation

$$
A_{\mathrm{f}, \tau}\left(\left(\boldsymbol{u}_{h}^{n-1}, p_{h}^{n-1}\right),\left(\mathcal{L}_{h} \boldsymbol{w}_{h}, 0\right)\right)
$$

Similarly, the discrete counterpart of $(6)_{2}$ corresponds to (9) with $q_{h}=0$. Finally, the discrete counterpart of $(7)$ is chosen as

$$
\int_{\Omega_{\mathrm{f}}} \operatorname{div} \boldsymbol{u}_{h}^{n} q_{h}+\frac{\gamma_{0}}{\gamma \mu} \int_{\Sigma_{\mathrm{f}, h}}\left(p_{h}^{n}-p_{h}^{n-1}\right) q_{h}=\int_{\Sigma_{\mathrm{f}, h}} \operatorname{div}\left(\mathcal{L}_{h}\left(\boldsymbol{u}_{h}^{n}-\dot{\boldsymbol{d}}_{h}^{n}\right)\right) q_{h} .
$$

Note that all these terms are made of volume integrals. In particular, the weakly consistent compressibility acts on the first layer of fluid (volume) elements $\Sigma_{\mathrm{f}, h}$, while in (5) it works only on the interface $\Sigma$. This explains the missing factor, $h$, in the compressibility scaling (actually hidden in the width of the layer $\Sigma_{\mathrm{f}, h}$ ). The derivation of (10) will be further elucidated in the stability analysis reported in the next section.

\subsubsection{Stability analysis.}

Below we will show that a stability analysis similar to that of [6] can be carried out for Algorithm 3. To handle the residual terms we shall need the following continuity estimates for the 
lifting operator $\mathcal{L}_{h}$ (see, e.g., [15] for a proof):

$$
\begin{aligned}
\left\|\mathcal{L}_{h} \boldsymbol{\xi}_{h}\right\|_{0, \Omega_{\mathrm{f}}} & \leq C_{\mathcal{L}} h^{\frac{1}{2}}\left\|\boldsymbol{\xi}_{h}\right\|_{0, \Sigma}, \\
\left\|\boldsymbol{\nabla} \mathcal{L}_{h} \boldsymbol{\xi}_{h}\right\|_{0, \Omega_{\mathrm{f}}} & \leq C_{\mathcal{L}} h^{-\frac{1}{2}}\left\|\boldsymbol{\xi}_{h}\right\|_{0, \Sigma}
\end{aligned}
$$

for all $\boldsymbol{\xi} \in \boldsymbol{V}_{h}(\Sigma)$. The next result states the conditional energy stability of Algorithm 3 .

Theorem 2 Assume that the fluid-structure system is isolated, i.e., $p_{\text {out }}=0$ and $\boldsymbol{u}_{\text {in }}=\mathbf{0}$. Let $\left\{\left(\boldsymbol{u}_{h}^{n}, p_{h}^{n}, \boldsymbol{d}_{h}^{n}, \dot{\boldsymbol{d}}_{h}^{n}\right)\right\}_{1 \leq n \leq N}$ be given by Algorithm 3. Then, under the following conditions

$$
\rho_{\mathrm{f}} C_{\mathcal{L}}^{2} h^{2} \lesssim \gamma \tau \mu, \quad \gamma \tau \lesssim h, \quad C_{\mathcal{L}}^{2} \lesssim \gamma_{0}, \gamma
$$

there holds

$$
E^{n} \lesssim E^{0}+\rho_{\mathrm{f}}\left\|\boldsymbol{u}_{h}^{0}-\boldsymbol{u}_{h}^{-1}\right\|_{0, \Omega_{\mathrm{f}}}^{2}+C_{\mathcal{L}}^{2} \mu\left\|\boldsymbol{u}_{h}^{0}\right\|_{0, \Sigma}^{2}+\mu\left\|\boldsymbol{\epsilon}\left(\boldsymbol{u}_{h}^{0}\right)\right\|_{0, \Omega_{\mathrm{f}}}^{2}+\frac{\gamma_{0} h}{\gamma \mu} \tau\left\|p_{h}^{0}\right\|_{0, \Sigma_{\mathrm{f}, h}}^{2}
$$

for all $1 \leq n \leq N$

Proof. Taking $\left(\boldsymbol{v}_{h}, q_{h}, \boldsymbol{w}_{h}, \boldsymbol{z}_{h}\right)=\tau\left(\boldsymbol{u}_{h}^{n}, p_{h}^{n}, \partial_{\tau} \boldsymbol{d}_{h}^{n}, \partial_{\tau} \dot{\boldsymbol{d}}_{h}^{n}\right)$ in (8)-(9) and adding the resulting expressions we get

$$
\begin{aligned}
E^{n}+\frac{\rho_{\mathrm{f}}}{2}\left\|\boldsymbol{u}_{h}^{n}-\boldsymbol{u}_{h}^{n-1}\right\|_{0, \Omega_{\mathrm{f}}}^{2}+2 \mu \tau\left\|\boldsymbol{\epsilon}\left(\boldsymbol{u}_{h}^{n}\right)\right\|_{0, \Omega_{\mathrm{f}}}^{2}+\frac{\gamma_{0} \tau}{2 \gamma \mu}\left\|p_{h}^{n}-p_{h}^{n-1}\right\|_{0, \Sigma_{\mathrm{f}, h}}^{2} & \\
& +\frac{\gamma_{0} \tau^{2}}{2 \gamma \mu} \partial_{\tau}\left\|p_{h}^{n}\right\|_{0, \Sigma_{\mathrm{f}, h}}^{2}+\frac{\gamma \mu \tau}{h} \underbrace{\left[\int_{\Sigma}\left(\boldsymbol{u}_{h}^{n}-\dot{\boldsymbol{d}}_{h}^{n}\right) \cdot \boldsymbol{u}_{h}^{n}+\int_{\Sigma}\left(\dot{\boldsymbol{d}}_{h}^{n}-\boldsymbol{u}_{h}^{n-1}\right) \cdot \dot{\boldsymbol{d}}_{h}^{n}\right]}_{T_{1}} \\
& -\tau \underbrace{\left[A_{\mathrm{f}, \tau}\left(\left(\boldsymbol{u}_{h}^{n-1}, p_{h}^{n-1}\right),\left(\mathcal{L}_{h}\left(\boldsymbol{u}_{h}^{n}-\dot{\boldsymbol{d}}_{h}^{n}\right), 0\right)\right)+\int_{\Sigma_{\mathrm{f}, h}} \boldsymbol{\nabla} \cdot \mathcal{L}_{h}\left(\boldsymbol{u}_{h}^{n}-\dot{\boldsymbol{d}}_{h}^{n}\right) p_{h}^{n}\right]}_{T_{2}}=0 .
\end{aligned}
$$

The first term is treated as in [6], which yields

$$
\begin{aligned}
T_{1} & =\left\|\boldsymbol{u}_{h}^{n}-\dot{\boldsymbol{d}}_{h}^{n}\right\|_{0, \Sigma}^{2}+\int_{\Sigma}\left(\boldsymbol{u}_{h}^{n}-\boldsymbol{u}_{h}^{n-1}\right) \cdot \dot{\boldsymbol{d}}_{h}^{n} \\
& =\left\|\boldsymbol{u}_{h}^{n}-\dot{\boldsymbol{d}}_{h}^{n}\right\|_{0, \Sigma}^{2}+\int_{\Sigma}\left(\boldsymbol{u}_{h}^{n}-\boldsymbol{u}_{h}^{n-1}\right) \cdot\left(\dot{\boldsymbol{d}}_{h}^{n}-\boldsymbol{u}_{h}^{n}\right)+\int_{\Sigma}\left(\boldsymbol{u}_{h}^{n}-\boldsymbol{u}_{h}^{n-1}\right) \cdot \boldsymbol{u}_{h}^{n} \\
& \geq \frac{1}{2}\left\|\boldsymbol{u}_{h}^{n}-\dot{\boldsymbol{d}}_{h}^{n}\right\|_{0, \Sigma}^{2}+\frac{\tau}{2} \partial_{\tau}\left\|\boldsymbol{u}_{h}^{n}\right\|_{0, \Sigma}^{2} \cdot
\end{aligned}
$$

Hence, (14) reduces to

$$
\begin{aligned}
E^{n}+\frac{\rho_{\mathrm{f}}}{2}\left\|\boldsymbol{u}_{h}^{n}-\boldsymbol{u}_{h}^{n-1}\right\|_{0, \Omega_{\mathrm{f}}}^{2}+2 \mu \tau\left\|\boldsymbol{\epsilon}\left(\boldsymbol{u}_{h}^{n}\right)\right\|_{0, \Omega_{\mathrm{f}}}^{2}+\frac{\gamma_{0} \tau}{2 \gamma \mu}\left\|p_{h}^{n}-p_{h}^{n-1}\right\|_{0, \Sigma_{\mathrm{f}, h}}^{2} \\
\quad+\frac{\gamma_{0} \tau^{2}}{2 \gamma \mu} \partial_{\tau}\left\|p_{h}^{n}\right\|_{0, \Sigma_{\mathrm{f}, h}}^{2}+\frac{\gamma \mu \tau}{2 h}\left\|\boldsymbol{u}_{h}^{n}-\dot{\boldsymbol{d}}_{h}^{n}\right\|_{0, \Sigma}^{2}+\frac{\gamma \mu \tau^{2}}{2 h} \partial_{\tau}\left\|\boldsymbol{u}_{h}^{n}\right\|_{0, \Sigma}^{2}+T_{2} \leq 0 .
\end{aligned}
$$


For the last term we first note that

$$
\begin{aligned}
& T_{2}=\underbrace{\int_{\Omega_{\mathrm{f}}} \partial_{\tau} \boldsymbol{u}_{h}^{n-1} \cdot \mathcal{L}_{h}\left(\boldsymbol{u}_{h}^{n}-\dot{\boldsymbol{d}}_{h}^{n}\right)}_{T_{2,1}}+\underbrace{2 \mu \int_{\Omega_{\mathrm{f}}} \boldsymbol{\epsilon}\left(\boldsymbol{u}_{h}^{n-1}\right): \boldsymbol{\epsilon}\left(\mathcal{L}_{h}\left(\boldsymbol{u}_{h}^{n}-\dot{\boldsymbol{d}}_{h}^{n}\right)\right)}_{T_{2,2}} \\
& +\underbrace{\int_{\Sigma_{\mathrm{f}, h}} \boldsymbol{\nabla} \cdot \mathcal{L}_{h}\left(\boldsymbol{u}_{h}^{n}-\dot{\boldsymbol{d}}_{h}^{n}\right)\left(p_{h}^{n}-p_{h}^{n-1}\right)}_{T_{2,3}} .
\end{aligned}
$$

Owing to (11), for the third term we have

$$
\begin{aligned}
T_{2,3} & \leq\left\|p_{h}^{n}-p_{h}^{n-1}\right\|_{0, \Sigma_{\mathrm{f}, h}}\left\|\nabla \cdot \mathcal{L}_{h}\left(\boldsymbol{u}_{h}^{n}-\dot{\boldsymbol{d}}_{h}^{n}\right)\right\|_{0, \Omega_{\mathrm{f}}} \\
& \leq\left\|p_{h}^{n}-p_{h}^{n-1}\right\|_{0, \Sigma_{\mathrm{f}, h}} C_{\mathcal{L}} h^{-\frac{1}{2}}\left\|\boldsymbol{u}_{h}^{n}-\dot{\boldsymbol{d}}_{h}^{n}\right\|_{0, \Sigma} \\
& \leq \frac{2 C_{\mathcal{L}}^{2}}{\gamma \mu}\left\|p_{h}^{n}-p_{h}^{n-1}\right\|_{0, \Sigma_{\mathrm{f}, h}}^{2}+\frac{\gamma \mu}{8 h}\left\|\boldsymbol{u}_{h}^{n}-\dot{\boldsymbol{d}}_{h}^{n}\right\|_{0, \Sigma}^{2} .
\end{aligned}
$$

Similarly, for the second we obtain

$$
\begin{aligned}
T_{2,2} & \leq 2 \mu\left\|\boldsymbol{\epsilon}\left(\boldsymbol{u}_{h}^{n-1}\right)\right\|_{0, \Omega_{\mathrm{f}}} \| \boldsymbol{\epsilon}\left(\mathcal{L}_{h}\left(\boldsymbol{u}_{h}^{n}-\dot{\boldsymbol{d}}_{h}^{n}\right) \|_{0, \Omega_{\mathrm{f}}}\right. \\
& \leq 2 \mu\left\|\boldsymbol{\epsilon}\left(\boldsymbol{u}_{h}^{n-1}\right)\right\|_{0, \Omega_{\mathrm{f}}} C_{\mathcal{L}} h^{-\frac{1}{2}}\left\|\boldsymbol{u}_{h}^{n}-\dot{\boldsymbol{d}}_{h}^{n}\right\|_{0, \Sigma} \\
& \leq \frac{8 C_{\mathcal{L}}^{2} \mu}{\gamma}\left\|\boldsymbol{\epsilon}\left(\boldsymbol{u}_{h}^{n-1}\right)\right\|_{0, \Omega_{\mathrm{f}}}^{2}+\frac{\gamma \mu}{8 h}\left\|\boldsymbol{u}_{h}^{n}-\dot{\boldsymbol{d}}_{h}^{n}\right\|_{0, \Sigma}^{2},
\end{aligned}
$$

and, for the first term

$$
\begin{aligned}
T_{2,1} & \leq \rho_{\mathrm{f}}\left\|\partial_{\tau} \boldsymbol{u}_{h}^{n-1}\right\|_{0, \Omega_{\mathrm{f}}}\left\|\mathcal{L}_{h}\left(\boldsymbol{u}_{h}^{n}-\dot{\boldsymbol{d}}_{h}^{n}\right)\right\|_{0, \Omega_{\mathrm{f}}} \\
& \leq \frac{\rho_{\mathrm{f}}}{\tau}\left\|\boldsymbol{u}_{h}^{n}-\boldsymbol{u}_{h}^{n-1}\right\|_{0, \Omega_{\mathrm{f}}} C_{\mathcal{L}} h^{\frac{1}{2}}\left\|\boldsymbol{u}_{h}^{n}-\dot{\boldsymbol{d}}_{h}^{n}\right\|_{0, \Sigma} \\
& \leq\left(\frac{2 \rho_{\mathrm{f}} C_{\mathcal{L}}^{2} h^{2}}{\gamma \mu \tau}\right) \frac{\rho_{\mathrm{f}}}{\tau}\left\|\boldsymbol{u}_{h}^{n}-\boldsymbol{u}_{h}^{n-1}\right\|_{0, \Omega_{\mathrm{f}}}^{2}+\frac{\gamma \mu}{8 h}\left\|\boldsymbol{u}_{h}^{n}-\dot{\boldsymbol{d}}_{h}^{n}\right\|_{0, \Sigma}^{2} .
\end{aligned}
$$

The estimate (13) then follows from (12) by inserting the last three bounds into (15) and then by summing the resulting expression over $m=1, \ldots, n$.

At this point it is worth recalling that the energy stability of the original Nitsche based explicit coupling (Algorithm 1) is independent of the choice of the time-marching in the fluid and in the structure. The above stability analysis, however, makes explicit use of the numerical dissipation provided by time-marching in the fluid (to control the first term in (16)). On the other hand no assumption is made on the time-marching for the solid (as for Algorithm 1).

By imposing the stronger inverse CFL-condition

$$
\rho_{\mathrm{f}} C_{\mathcal{L}}^{2} h^{2} \lesssim \gamma \tau^{2} \mu
$$

in the right hand side of equation (16) and then applying Gronwall's lemma, we can avoid the use of the numerical dissipation provided by the fluid time-marching. The main drawback of this approach is that the combination of the strengthened CFL-condition (17) with $\gamma \tau \lesssim h$ and $C_{\mathcal{L}}^{2} \lesssim \gamma_{0}$, leads to stability conditions depending on the physical parameters. 


\subsection{Genuine Robin-Robin explicit coupling}

The Robin-Robin schemes given by Algorithms 2 and 3, are not completely satisfactory since they still involve some non-standard terms. In particular, it would be advantageous if the terms

$$
\int_{\Sigma}\left(\boldsymbol{u}_{h}^{n}-\dot{\boldsymbol{d}}_{h}^{n}\right) \cdot \boldsymbol{n} q_{h}+\frac{\gamma_{0} h}{\gamma \mu} \int_{\Sigma}\left(p_{h}^{n}-p_{h}^{n-1}\right) q_{h}
$$

and

$$
\int_{\Sigma_{\mathrm{f}, h}} \operatorname{div}\left(\mathcal{L}_{h}\left(\boldsymbol{u}_{h}^{n}-\dot{\boldsymbol{d}}_{h}^{n}\right)\right) q_{h}+\frac{\gamma_{0}}{\gamma \mu} \int_{\Sigma_{\mathrm{f}, h}}\left(p_{h}^{n}-p_{h}^{n-1}\right) q_{h},
$$

could be omitted in Algorithms 2 and 3, respectively. The first term in (18) and (19) adds weak control of the difference $\left.\left(\boldsymbol{u}_{h}^{n}-\dot{\boldsymbol{d}}_{h}^{n}\right) \cdot \boldsymbol{n}\right|_{\Sigma}$, that is already controlled (at least for sufficiently viscous flows) by the Nitsche penalty term and the other term is the interface pressure penalty term that is introduced to stabilize the pressure fluctuations in time. We will now suggest a reduced coupling scheme, reported in Algorithm 4, where these terms are eliminated. Thus, instead of (7), the standard form of the continuity constraint, $\operatorname{div} \boldsymbol{u}^{n}=0$, is considered. Note that this method is nothing but the fully discrete counterpart of (1), (2) and (6), hence the terminology "genuine Robin-Robin". Although the stability result of the previous section does not extend to this case, the numerical experiments reported in Section 5 indicate that this method is as stable and accurate as the ones of Algorithms 1-3.

Algorithm 4 Genuine Robin-Robin explicit coupling scheme.

1. Solid sub-problem: find $\boldsymbol{d}_{h}^{n}, \dot{\boldsymbol{d}}_{h}^{n} \in \boldsymbol{V}_{\mathrm{s}, h}$ such that

$$
A_{\mathrm{s}, \tau}\left(\left(\boldsymbol{d}_{h}^{n}, \dot{\boldsymbol{d}}_{h}^{n}\right),\left(\boldsymbol{w}_{h}, \boldsymbol{z}_{h}\right)\right)+\gamma \frac{\mu}{h} \int_{\Sigma} \dot{\boldsymbol{d}}_{h}^{n} \cdot \boldsymbol{w}_{h}=\gamma \frac{\mu}{h} \int_{\Sigma} \boldsymbol{u}_{h}^{n} \cdot \boldsymbol{w}_{h}-\int_{\Sigma} \boldsymbol{\sigma}\left(\boldsymbol{u}_{h}^{n-1}, p_{h}^{n-1}\right) \boldsymbol{n} \cdot \boldsymbol{w}_{h}
$$

for all $\boldsymbol{w}_{h}, \boldsymbol{z}_{h} \in \boldsymbol{V}_{\mathrm{s}, h}$.

2. Fluid sub-problem: find $\left(\boldsymbol{u}_{h}^{n}, p_{h}^{n}\right) \in \boldsymbol{V}_{\mathrm{f}, h} \times Q_{h}$, with $\left.\boldsymbol{u}_{h}^{n}\right|_{\Gamma^{\text {in }}}=\boldsymbol{u}_{\mathrm{in}}^{n}$, such that

$$
\begin{array}{r}
A_{\mathrm{f}, \tau}\left(\left(\boldsymbol{u}_{h}^{n}, p_{h}^{n}\right),\left(\boldsymbol{v}_{h}, q_{h}\right)\right)+\gamma \frac{\mu}{h} \int_{\Sigma} \boldsymbol{u}_{h}^{n} \cdot \boldsymbol{v}_{h}=\gamma \frac{\mu}{h} \int_{\Sigma} \dot{\boldsymbol{d}}_{h}^{n} \cdot \boldsymbol{v}_{h}+\int_{\Sigma} \boldsymbol{\sigma}\left(\boldsymbol{u}_{h}^{n-1}, p_{h}^{n-1}\right) \boldsymbol{n} \cdot \boldsymbol{w}_{h} \\
-\int_{\Gamma \text { out }} p_{\text {out }}^{n} \boldsymbol{v}_{h} \cdot \boldsymbol{n}
\end{array}
$$

for all $\left(\boldsymbol{v}_{h}, q_{h}\right) \in \boldsymbol{V}_{\mathrm{f}, \Gamma^{\mathrm{in}}, h} \times Q_{h}$.

\section{Enhancing accuracy}

The splitting error is the main source of error in the explicit coupling schemes. This error appears both in the consistency terms on the interface and in the penalty term. The pressure stabilization on the boundary also introduces a consistency error but, as was noted in the introduction, this error is of higher order than the consistency error in the penalty terms. One way of reducing the splitting error is to introduce an extrapolated quantity in the coupling terms. This increases the formal order of the scheme, but may have negative influence on the stability properties. It is therefore often helpful to combine the extrapolation with a correction step. 
The largest consistency error is due to the penalty term on the jump of the velocities over the interface. This term can be omitted if the non-symmetric version of Nitsche's method is used. The stability of this scheme, without penalty, in the case of imposition of boundary conditions for Poisson's equation, was recently shown in [5] and for elasticity in [4].

\subsection{Second-order extrapolation with first-order correction}

To enhance the accuracy of Algorithm 1, we need to make the term $\gamma \frac{\mu}{h} \int_{\Sigma} \boldsymbol{u}_{h}^{n-1} \cdot \boldsymbol{w}_{h}$ closer to $\gamma \frac{\mu}{h} \int_{\Sigma} \boldsymbol{u}_{h}^{n} \cdot \boldsymbol{w}_{h}$. Indeed the choice of $\boldsymbol{u}_{h}^{n-1}$ leads to the following consistency perturbation compared to the implicit method:

$$
\gamma \frac{\mu}{h} \int_{\Sigma}\left(\dot{\boldsymbol{d}}_{h}^{n}-\boldsymbol{u}_{h}^{n-1}\right) \cdot \boldsymbol{w}_{h}=\gamma \frac{\mu}{h} \int_{\Sigma}\left(\dot{\boldsymbol{d}}_{h}^{n}-\boldsymbol{u}_{h}^{n}\right) \cdot \boldsymbol{w}_{h}+\gamma \frac{\mu \tau}{h} \int_{\Sigma} \partial_{t} \boldsymbol{u}_{h}(\tilde{\tau}) \cdot \boldsymbol{w}_{h},
$$

for some $\tilde{\tau} \in\left[t_{n-1}, t_{n}\right]$. The simplest way to achieve this is, for $n \geq 2$ to replace the term $\gamma \frac{\mu}{h} \int_{\Sigma} \boldsymbol{u}_{h}^{n-1} \cdot \boldsymbol{w}_{h}$ in (4) by $\gamma \frac{\mu}{h} \int_{\Sigma} \boldsymbol{u}_{h}^{*} \cdot \boldsymbol{w}_{h}$, where $\boldsymbol{u}_{h}^{*}$ is chosen as a second order extrapolation, typically $\boldsymbol{u}_{h}^{*}=2 \boldsymbol{u}_{h}^{n-1}-\boldsymbol{u}_{h}^{n-2}$. The idea behind the extrapolation is to obtain

$$
\gamma \frac{\mu}{h} \int_{\Sigma}\left(\dot{\boldsymbol{d}}_{h}^{n}-\boldsymbol{u}_{h}^{*}\right) \cdot \boldsymbol{w}_{h}=\gamma \frac{\mu}{h} \int_{\Sigma}\left(\dot{\boldsymbol{d}}_{h}^{n}-\boldsymbol{u}_{h}^{n}\right) \cdot \boldsymbol{w}_{h}+\gamma \frac{\mu \tau^{2}}{h} \int_{\Sigma} \partial_{t t} \boldsymbol{u}_{h}(\tilde{\tau}) \cdot \boldsymbol{w}_{h},
$$

for some $\tilde{\tau} \in\left[t_{n-2}, t_{n}\right]$. This results in an improvement in the formal error from $\tau / h$ to $\tau^{2} / h$, or from $(\tau / h)^{k+1}$ to $\left(\tau^{2} / h\right)^{k+1}$ if $k$ corrections are used, that is, if steps 1 and 2 of the scheme are solved iteratively $k+1$ times using the data from the previous iteration (see [6, Section 5.4]). Then the choice $\tau=O(h)$, imposed by the stability theorem leads to the error contributions $h$, for $k=0$ and $h^{2}$ for $k=1$, which is satisfactory for affine finite elements and second order time-discretization.

Numerical experimentation shows that the numerical scheme using extrapolation is unstable for the base case $k=0$. If on the other hand one or more correction steps are used $(k \geq 1)$ the method is stable and the expected orders are observed. This will be illustrated in the numerical experiments of Section 5. A potential disadvantage of this approach is that depending on the mesh parameters, more than one correction may be needed to ensure robustness.

\subsection{Non-symmetric penalty-free formulation}

If the non-symmetric Nitsche method is used, the penalty term on the velocities over the interface may be dropped without loss of stability. The accuracy in the $H^{1}$-norm is also the same, but the error constant is often slightly larger than for the penalized formulation (provided a close to optimal choice of the penalty parameter is made). We give the algorithm of the explicit coupling scheme resulting from the use of the non-symmetric penalty-free formulation in Algorithm 5 .

The interpretation of this scheme is that of a Neumann/Dirichlet coupling, where forces are transmitted to the solid using a Neumann condition and a weak Dirichlet condition couples the fluid velocities to the solid ones. The weak Dirichlet condition can be interpreted as a Lagrangemultiplier coupling where the Lagrange multiplier spaces consists of the space of normal fluxes constructed using the finite element spaces $V_{\mathrm{f}, \Gamma^{\mathrm{in}}, h} \times Q_{h}$ on the interface. Note that the part $\boldsymbol{\sigma}\left(\boldsymbol{v}_{h}, 0\right) \boldsymbol{n}$ corresponds to the imposition of the constraints due to the viscous forces and $\boldsymbol{\sigma}\left(0, q_{h}\right) \boldsymbol{n}$ corresponds to the imposition of the non-penetration condition.

When applying an explicit coupling, the inconsistency in time due to the penalty term vanishes, which leads to a method which has first order accuracy in time without any extrapolation/correction steps. 
$\overline{\text { Algorithm } 5 \text { Stabilized explicit coupling using a penalty free non-symmetric Nitsche type }}$ method.

1. Solid sub-problem: find $\boldsymbol{d}_{h}^{n}, \dot{\boldsymbol{d}}_{h}^{n} \in \boldsymbol{V}_{\mathrm{s}, h}$ such that

$$
A_{\mathrm{s}, \tau}\left(\left(\boldsymbol{d}_{h}^{n}, \dot{\boldsymbol{d}}_{h}^{n}\right),\left(\boldsymbol{w}_{h}, \boldsymbol{z}_{h}\right)\right)=-\int_{\Sigma} \boldsymbol{\sigma}\left(\boldsymbol{u}_{h}^{n-1}, p_{h}^{n-1}\right) \boldsymbol{n} \cdot \boldsymbol{w}_{h}
$$

for all $\boldsymbol{w}_{h}, \boldsymbol{z}_{h} \in \boldsymbol{V}_{\mathrm{s}, h}$.

2. Fluid sub-problem: find $\left(\boldsymbol{u}_{h}^{n}, p_{h}^{n}\right) \in \boldsymbol{V}_{\mathrm{f}, h} \times Q_{h}$, with $\left.\boldsymbol{u}_{h}^{n}\right|_{\Gamma^{\text {in }}}=\boldsymbol{u}_{\text {in }}^{n}$, such that

$$
\begin{aligned}
A_{\mathrm{f}, \tau}\left(\left(\boldsymbol{u}_{h}^{n}, p_{h}^{n}\right),\left(\boldsymbol{v}_{h}, q_{h}\right)\right)+ & \int_{\Sigma} \boldsymbol{u}_{h}^{n} \cdot \boldsymbol{\sigma}\left(\boldsymbol{v}_{h}, q_{h}\right) \boldsymbol{n}+\frac{\gamma_{0} h}{\mu} \int_{\Sigma} p_{h}^{n} q_{h}=\frac{\gamma_{0} h}{\mu} \int_{\Sigma} p_{h}^{n-1} q_{h} \\
& +\int_{\Sigma} \dot{\boldsymbol{d}}_{h}^{n} \cdot \boldsymbol{\sigma}\left(\boldsymbol{v}_{h}, q_{h}\right) \boldsymbol{n}+\int_{\Sigma} \boldsymbol{\sigma}\left(\boldsymbol{u}_{h}^{*}, p_{h}^{*}\right) \boldsymbol{n} \cdot \boldsymbol{v}_{h}-\int_{\Gamma_{\text {out }}} p_{\text {out }}^{n} \boldsymbol{v}_{h} \cdot \boldsymbol{n}
\end{aligned}
$$

for all $\left(\boldsymbol{v}_{h}, q_{h}\right) \in \boldsymbol{V}_{\mathrm{f}, \Gamma^{\mathrm{in}}, h} \times Q_{h}$. Here either $\left(\boldsymbol{u}_{h}^{*}, p_{h}^{*}\right)=\left(\boldsymbol{u}_{h}^{n-1}, p_{h}^{n-1}\right)$ or $\left(\boldsymbol{u}_{h}^{*}, p_{h}^{*}\right)=\left(\boldsymbol{u}_{h}^{n}, p_{h}^{n}\right)$.

Note that two choices are possible for the consistency part of the interface fluid stresses, $\int_{\Sigma} \boldsymbol{\sigma}\left(\boldsymbol{u}_{h}^{*}, p_{h}^{*}\right) \boldsymbol{n} \cdot \boldsymbol{v}_{h}$, either $\left(\boldsymbol{u}_{h}^{*}, p_{h}^{*}\right)=\left(\boldsymbol{u}_{h}^{n-1}, p_{h}^{n-1}\right)$ or $\left(\boldsymbol{u}_{h}^{*}, p_{h}^{*}\right)=\left(\boldsymbol{u}_{h}^{n}, p_{h}^{n}\right)$. In our numerical experiments both approaches were stable and first order convergent in two-space dimensions. In the three dimensional case however, the linear systems of the former case proved too ill-conditioned for the iterative solver. Below we only present the results obtained with the more robust second method. The main difference between the methods is in the error constant which is approximately $50 \%$ larger for the second method. As will be shown in the numerical section, in general, the error constant is larger for the non-symmetric method (Algorithm 5) compared to the penalty based methods (Algorithms 1-4). On the other hand, in our numerical experiments, the explicit coupling scheme using the non-symmetric version proved to be stable and first order convergent without resorting to extrapolation or correction steps, which makes the method cheaper for a fixed time-step/mesh combination.

\subsection{Penalty free explicit coupling scheme using the non-symmetric Nitsche method without pressure stabilization}

It is tempting to combine the ideas of Section 3.3 and Section 4.2, into a method where the penalty on the velocity jump across the interface is eliminated by the use of the non-symmetric version of Nitsche's method (with $\left(u_{h}^{*}, p_{h}^{*}\right)=\left(u_{h}^{n-1}, p_{h}^{n-1}\right)$ and the pressure stabilization is eliminated by the omission of the term $\int_{\Sigma}\left(\boldsymbol{u}_{h}^{n}-\dot{\boldsymbol{d}}_{h}^{n}\right) \cdot \boldsymbol{\sigma}\left(0, q_{h}\right) \boldsymbol{n}$ as was proposed in Section 3.3. In Algorithm 6 we propose such a method. Numerical investigations indicate that the resulting scheme indeed is stable, however since the non-penetration condition has been relaxed by the omission of the corresponding term, the coupling relies solely on the interface term $\int_{\Sigma}\left(\boldsymbol{u}_{h}^{n}-\dot{\boldsymbol{d}}_{h}^{n}\right) \cdot \boldsymbol{\sigma}\left(\boldsymbol{v}_{h}, 0\right) \boldsymbol{n}$, which will scale linearly in the viscosity $\mu$. Hence even for moderately small viscosities the control of the non-penetration condition is dramatically reduced. Our numerical investigations (not reported here) indicate that this has an immediate effect on the computational accuracy. To sum up: the fully penalty-free method is stable and appears to be convergent, but the constant in the error estimate constant scales as $\mu^{-1}$, leading to a numerical method that is not competitive in practice. For small values of $\mu$ a similar effect is present in the method of Section 3.3, but in this 
Algorithm 6 Stabilized explicit coupling using penalty free non-symmetric Nitsche type method.

1. Solid sub-problem: find $\boldsymbol{d}_{h}^{n}, \dot{\boldsymbol{d}}_{h}^{n} \in \boldsymbol{V}_{\mathrm{s}, h}$ such that

$$
A_{\mathrm{s}, \tau}\left(\left(\boldsymbol{d}_{h}^{n}, \dot{\boldsymbol{d}}_{h}^{n}\right),\left(\boldsymbol{w}_{h}, \boldsymbol{z}_{h}\right)\right)=-\int_{\Sigma} \boldsymbol{\sigma}\left(\boldsymbol{u}_{h}^{n-1}, p_{h}^{n-1}\right) \boldsymbol{n} \cdot \boldsymbol{w}_{h}
$$

for all $\boldsymbol{w}_{h}, \boldsymbol{z}_{h} \in \boldsymbol{V}_{\mathrm{s}, h}$

2. Fluid sub-problem: find $\left(\boldsymbol{u}_{h}^{n}, p_{h}^{n}\right) \in \boldsymbol{V}_{\mathrm{f}, h} \times Q_{h}$, with $\left.\boldsymbol{u}_{h}^{n}\right|_{\Gamma^{\text {in }}}=\boldsymbol{u}_{\text {in }}^{n}$, such that

$$
\begin{aligned}
A_{\mathrm{f}, \tau}\left(\left(\boldsymbol{u}_{h}^{n}, p_{h}^{n}\right),\left(\boldsymbol{v}_{h}, q_{h}\right)\right) & +\int_{\Sigma} \boldsymbol{u}_{h}^{n} \cdot 2 \mu \boldsymbol{\epsilon}\left(\boldsymbol{v}_{h}\right) \boldsymbol{n}= \\
& +\int_{\Sigma} \boldsymbol{\sigma}\left(\boldsymbol{u}_{h}^{n-1}, p_{h}^{n-1}\right) \boldsymbol{n} \cdot \boldsymbol{v}_{h}+\int_{\Sigma} \dot{\boldsymbol{d}}_{h}^{n} \cdot 2 \mu \boldsymbol{\epsilon}\left(\boldsymbol{v}_{h}\right) \boldsymbol{n}-\int_{\Gamma_{\text {out }}} p_{\text {out }}^{n} \boldsymbol{v}_{h} \cdot \boldsymbol{n}
\end{aligned}
$$

for all $\left(\boldsymbol{v}_{h}, q_{h}\right) \in \boldsymbol{V}_{\mathrm{f}, \Gamma^{\text {in }}, h} \times Q_{h}$.

case the problem can be solved by choosing the velocity penalty parameter sufficiently large.

\section{Numerical investigations}

Here we will give some illustration of the performance of the various methods presented above. In particular we will show that the formal orders discussed in the beginning are relevant and that the explicit splitting scheme of Algorithm 1 alone is insufficient to obtain optimal accuracy under the hyperbolic CFL. The approach using extrapolation and defect correction yields optimal convergence under hyperbolic CFL, as does the non-symmetric method of Algorithm 5 automatically, without resorting to any correction. We first give a detailed convergence study of a two-dimensional test case where the approximations are compared to a reference solution computed with an implicit coupling scheme on a very fine space-time grid (fully conformal spatial discretization). Here we investigate the convergence properties of Algorithms 2, 4 and 5. In the three dimensional case, we illustrate the accuracy of the methods in two fluid-structure examples from the literature.

\subsection{Convergence study in a two-dimensional benchmark}

In order to illustrate the accuracy of the proposed schemes, we have performed a series of numerical tests in a two-dimensional IFSI benchmark (see, e.g., [2]). We consider the coupled problem (1)-(3) with the fluid and solid domains given, respectively, by the rectangles $\Omega^{\mathrm{f}}=[0,6] \times[0,0.5]$ and $\Omega^{\mathrm{s}}=[0,6] \times[0.5,0.6]$. The interface is $\Sigma=[0,6] \times\{0.5\}$ and we have $\Gamma^{\mathrm{d}}=\{0,6\} \times[0.5,0.6]$, $\Gamma^{\mathrm{n}}=[0,6] \times\{0.6\}, \Gamma^{\mathrm{in}}=\{0\} \times[0,0.5]$ and $\Gamma^{\text {out }}=\{6\} \times[0,0.5]$. All units are in the CGS system. A sinusoidal pressure of maximal amplitude $2 \times 10^{4}$ is imposed in $\Gamma^{\text {in }}$ during $5 \times 10^{-3} \mathrm{~s}$, corresponding to half a period. Zero pressure is enforced on $\Gamma^{\text {out }}$ and a symmetry condition is applied on the lower wall $y=0$. The fluid physical parameters are given by $\rho^{\mathrm{f}}=1.0, \mu=0.035$. For the solid we have $\rho^{\mathrm{s}}=1.1, L_{1}=1.15 \times 10^{6}, L_{2}=1.7 \times 10^{6}, \beta=4 \times 10^{6}$. Affine finite elements are used for both the fluid and the structure (a pressure-stabilized formulation is considered in the fluid). The computations have been performed with FreeFem $++[35]$. For illustration purposes, we have reported in in Figure 3 some snapshots of the fluid pressure and deformed fluid and 

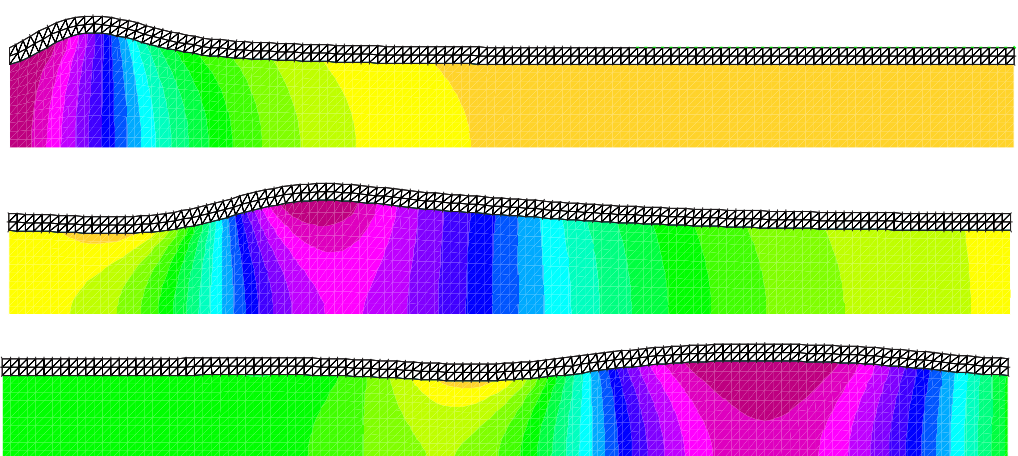

Figure 3: Snapshots of the fluid pressure and solid displacement at $t=0.003,0.008,0.015$ (from top to bottom). Algorithm 2 with second-order extrapolation and first-order correction, $\gamma_{0}=1$, $\gamma=1000, \tau=10^{-4}$ and $h=0.05$.

solid domains, obtained with Algorithm $2\left(\gamma_{0}=1, \gamma=1000\right)$ and the extrapolation/correction procedure of Section 4.1.

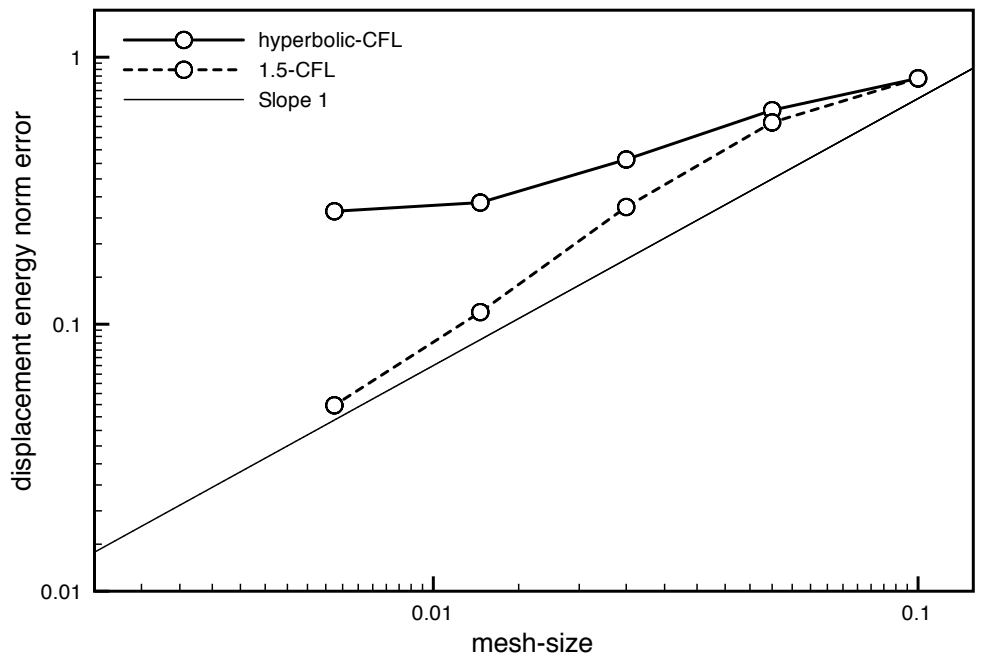

Figure 4: Solid displacement error convergence histories (in the relative elastic energy-norm) at time $t=0.015$, obtained from Algorithm 2 with one correction, for $\gamma_{0}=1, \gamma=1000, \tau=\mathcal{O}(h)$ (hyperbolic-CFL) and $\tau=\mathcal{O}\left(h^{\frac{3}{2}}\right)(1.5$-CFL).

A reference solution has been generated using a fully implicit coupling scheme and a high grid resolution $\left(\tau=10^{-6}\right.$ and $\left.h=3.125 \times 10^{-3}\right)$. We have first refined both in time and in space at the same rate, with the following set of discrete parameters:

$$
(\tau, h) \in\left\{\left(\frac{5 \times 10^{-4}}{2^{i}}, \frac{10^{-1}}{2^{i}}\right)\right\}_{i=0}^{4} .
$$

Note that this allows to highlight the $h$-uniformity of the convergence in time. 


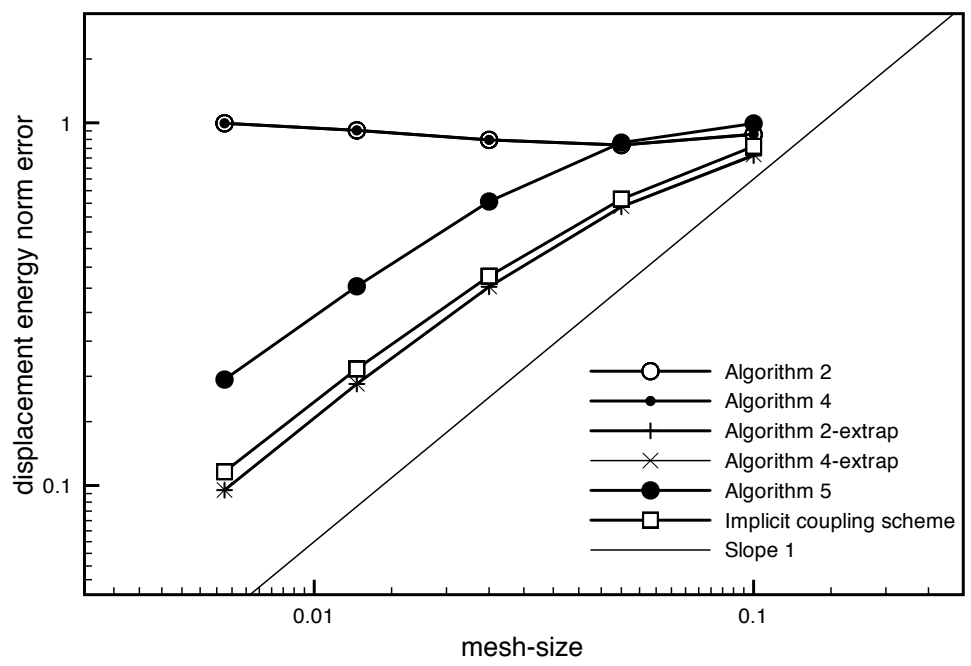

Figure 5: Solid displacement error convergence histories (in the relative elastic energy-norm) at time $t=0.015$ with $\tau=\mathcal{O}(h)$.

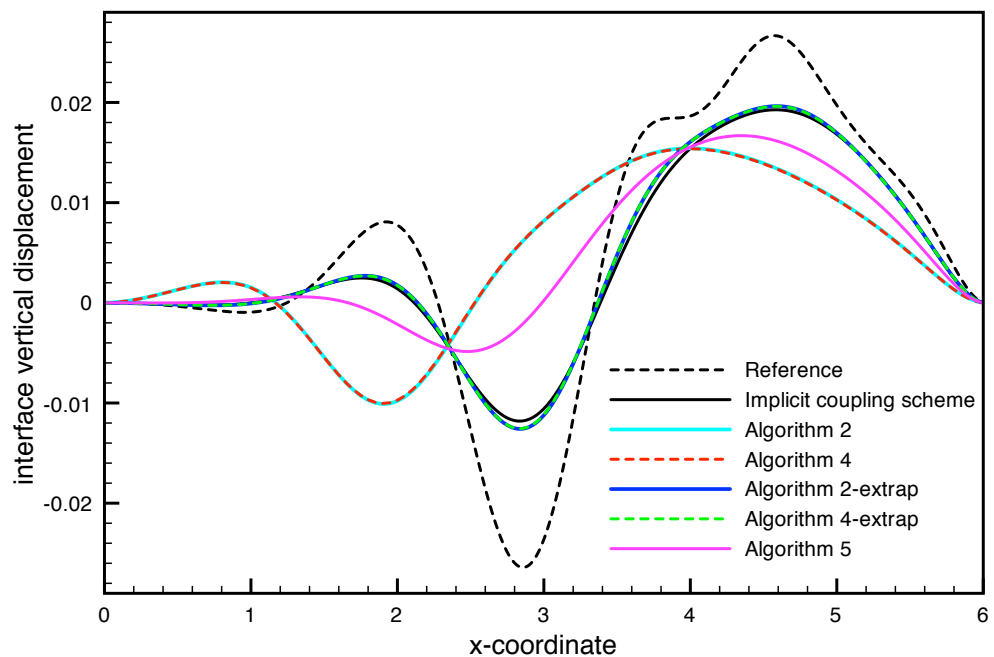

Figure 6: Comparison of the interface displacement at time $t=0.015$ obtained with $(\tau, h)=$ $\left(5 \times 10^{-4}, 10^{-1}\right) / 4$.

In order to illustrate the $\mathcal{O}\left((\tau / h)^{2}\right)$ error perturbation introduced by the explicit treatment in Algorithm 2 with one correction iteration, we present in Figure 4 the convergence histories of the solid displacement error obtained with $\tau=\mathcal{O}(h)$ (hyperbolic-CFL) and $\tau=\mathcal{O}\left(h^{\frac{3}{2}}\right)(1.5$ CFL). The expected non-convergent behavior under the hyperbolic-CFL constraint is clearly visible. The figure also shows that overall $\mathcal{O}(h)$ first-order accuracy can be achieved trough the strengthened CFL constraint $\tau=\mathcal{O}\left(h^{\frac{3}{2}}\right)$. 


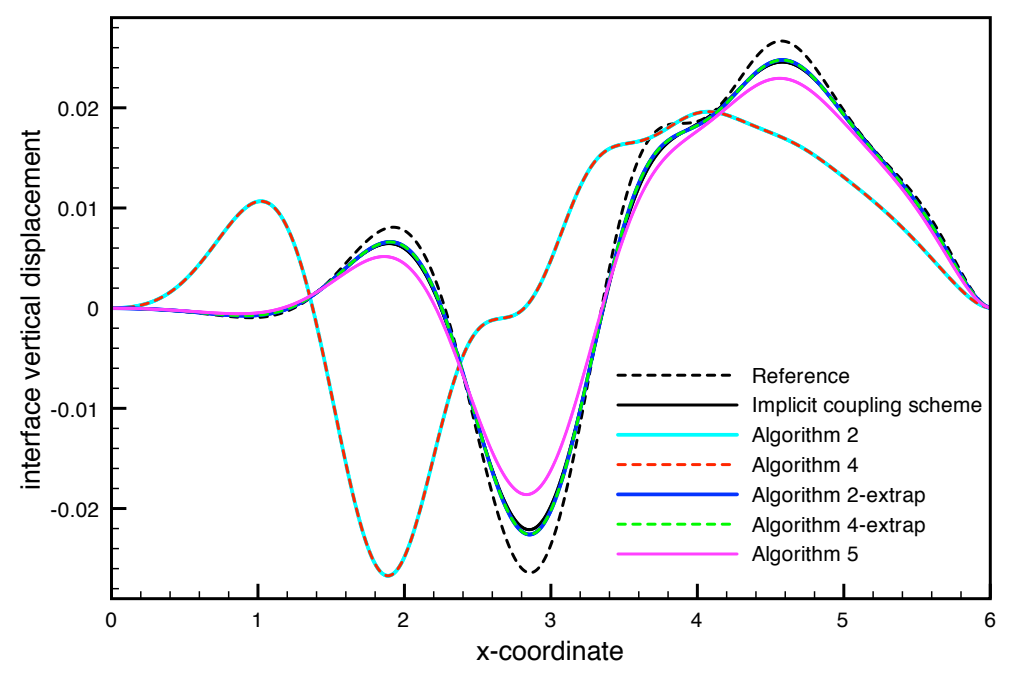

Figure 7: Comparison of the interface displacement at time $t=0.015$ obtained with $(\tau, h)=$ $\left(5 \times 10^{-4}, 10^{-1}\right) / 16$.

We now turn our attention to the accuracy of the schemes introduced in Section 4 under the standard hyperbolic-CFL constraint $\tau=\mathcal{O}(h)$. Figure 5 reports the convergence histories obtained for computations of approximations of the model problem using Algorithms 2, 4 (with $\gamma_{0}=1, \gamma=1000$ ) and Algorithm 5 (with $\left(\boldsymbol{u}_{h}^{*}, p_{h}^{*}\right)=\left(\boldsymbol{u}_{h}^{n}, p_{h}^{n}\right)$ and $\left.\gamma_{0}=0.005\right)$. For reference we also give the convergence of the implicit coupling scheme. Comparing first the purely explicit schemes where neither extrapolation nor correction steps are used (Algorithms 2, 4 and 5) we see that the only method that converges is the one given by Algorithm 5 . Both Algorithms 2 and 4 stagnate due to the truncation error from the penalty term. If on the other hand the extrapolation/correction procedure of Section 4.1 is applied to Algorithms 2 and 4 they perform as well as the implicit method. In all the cases we considered here, extrapolation followed by one correction step was sufficient to recover optimal convergence without robustness issues. During numerical experimentation, not reported, we did however observe that finer meshes may require a moderate increase in the number of correction steps. All the methods seem to have similar first order convergence. The error constant in the curve corresponding to the solutions produced using Algorithm 5 is approximately a factor two larger than for the other methods. Note however that the method of Algorithm 5 does not use any extrapolation or correction steps and is therefore cheaper for a fixed combination $h$ and $\tau$. Numerical experiments not reported here show that the constant can be reduced by adding a correction term or by using an explicit treatment of the fluid boundary stresses (i.e., $\left.\left(\boldsymbol{u}_{h}^{*}, p_{h}^{*}\right)=\left(\boldsymbol{u}_{h}^{n-1}, p_{h}^{n-1}\right)\right)$.

The impact of the error constant is illustrated in Figures 6 and 7, where we have depicted the final interface displacements obtained with the set of discretization parameters given by $i=2$ and $i=4$ in (20). For the finest space-time resolution, Algorithm 5 and Algorithms 2, 4 with extrapolation/correction yield similar accuracy as the implicit coupling scheme, while the quality of the approximations provided by Algorithm 5 degrades for the coarsest discretizations. The non-convergent behavior of Algorithms 2, 4 without extrapolation/correction is also clearly visible. 


\subsection{Three-dimensional test cases}

Comparisons are made with a reference solution obtained with an implicit coupling scheme (solved through a partitioned Dirichlet-Neumann interface Newton-GMRES algorithm, see e.g. [16]) and a fully conformal spatial discretization.

\subsubsection{Pressure wave propagation in a straight cylindrical vessel.}

In order to asses the proposed algorithms in their non-linear versions we considered threedimensional example proposed in [19] (see also [20, Chapter 12]). The fluid domain is a straight tube of radius 0.5 and of length 5 . All units are in the CGS system. The fluid is governed by the incompressible Navier-Stokes equations in ALE formulation. The vessel wall has a thickness of 0.1 and is clamped at its extremities. The vessel displacement is described by the linear elasticity equations.
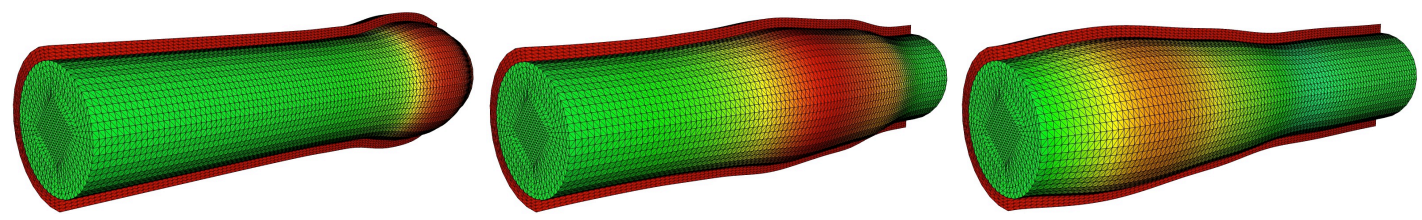

Figure 8: Snapshots of the fluid pressure and solid deformation at $t=0.003,0.007,0.011$ (from left to right) obtained with Algorithm $3\left(\gamma_{0}=1, \gamma=2500\right)$ with the second-order extrapolation and the first-order correction.

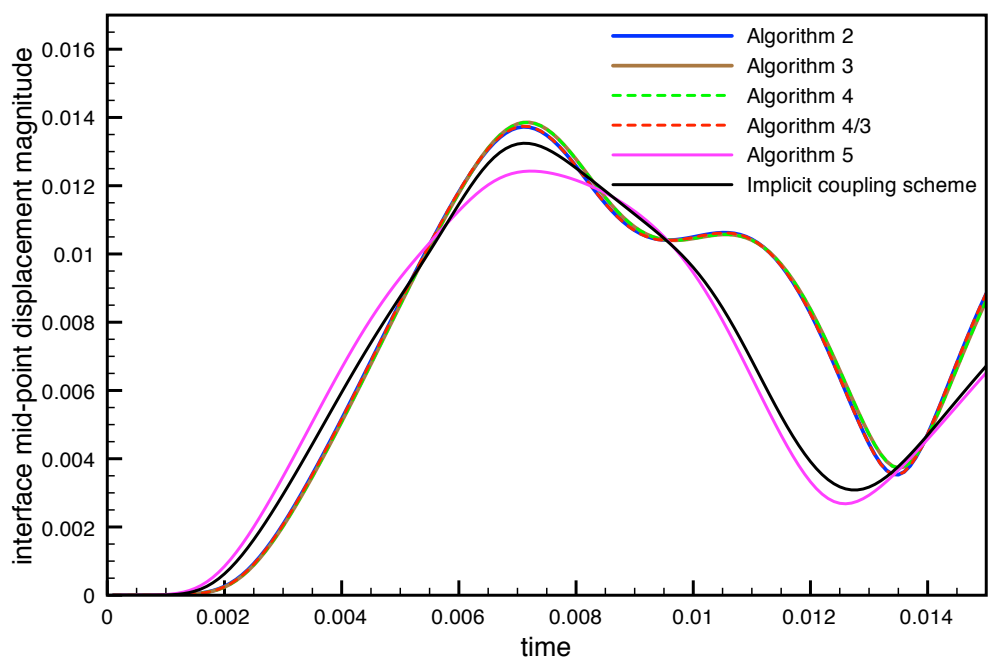

Figure 9: Comparison of the implicit, the Robin-Robin explicit coupling schemes without secondorder extrapolation and Algorithm 5: interface mid-point displacement magnitude vs. time.

The physical parameters for the fluid have been chosen as $\rho^{\mathrm{f}}=1$ and $\mu=0.035$. For the solid we have $\rho^{\mathrm{s}}=1.2$, Young modulus $E=3 \times 10^{6}$ and Poisson's ratio $\nu=0.3$. The overall system is initially at rest and, during the first $5 \times 10^{-3}$, an over pressure of $1.3332 \times 10^{4}$ is imposed 


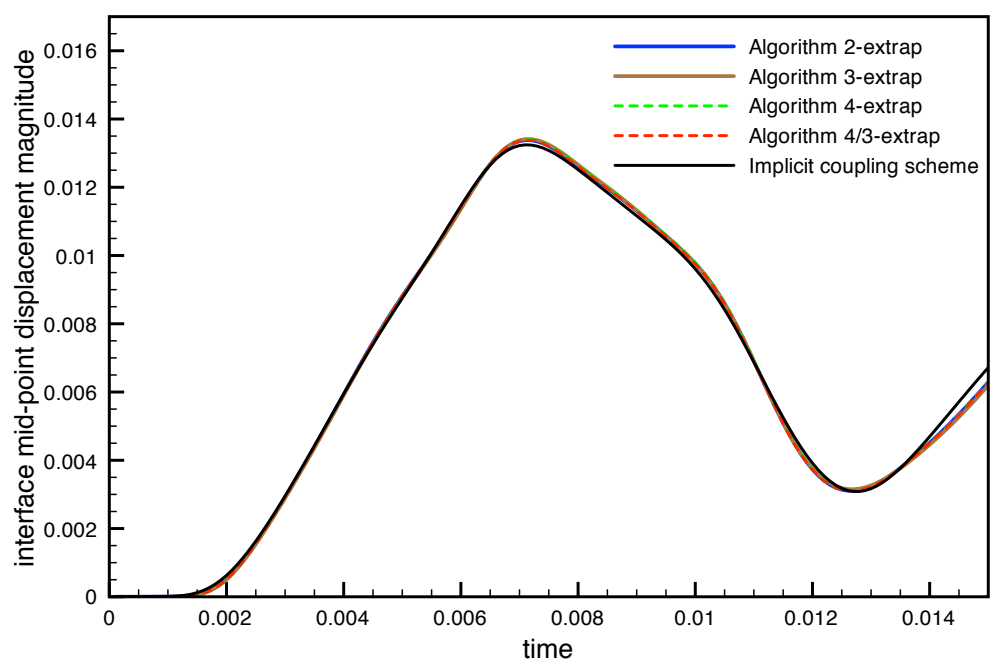

Figure 10: Comparison of the implicit and Robin-Robin explicit coupling schemes with secondorder extrapolation: interface mid-point displacement magnitude $v s$. time.

on the inlet boundary. Simulations are carried out on 150 time-steps of size $\tau=10^{-4}$. We have reported in in Figure 8 some snapshots of the fluid pressure and deformed fluid and solid domains, obtained with Algorithm $3\left(\gamma_{0}=1, \gamma=2500\right)$ with the second-order extrapolation and the first-order correction (see Section 4.1). The propagation of the pressure wave is clearly visible (for illustration purposes, the deformation of the fluid and solid domains has been magnified by a factor 10).

In this example we consider Algorithm 2, Algorithm 3, Algorithm 4 and Algorithm 5 (with $\left.\left(\boldsymbol{u}_{h}^{*}, p_{h}^{*}\right)=\left(\boldsymbol{u}_{h}^{n}, p_{h}^{n}\right)\right)$. We also investigate the genuine Robin-Robin scheme (Algorithm 4) with a residual based treatment of the coupling (as in Algorithm 3). In what follows, this scheme is termed Algorithm 4/3. Figure 9 reports the interface mid-point displacement magnitudes obtained with the Robin-Robin schemes (without second-order extrapolation and first-order correction), Algorithm 5 and the implicit coupling. The results provided by the Robin-Robin schemes are practically indistinguishable. Their mismatch with the implicit coupling scheme is however noticeable. On the other hand, Algorithm 5 is the only method which provides a physically correct solution without resorting to corrections. In Figure 10, we have reported the results obtained with the Robin-Robin schemes using a second-order extrapolation and the first-order correction. The second order-extrapolation retrieves the accuracy of the implicit coupling scheme.

\subsubsection{A physiological test-case.}

We consider now the numerical fluid-structure simulations reported in [1] using in vitro aneurysm geometries (see $[38,37])$. The fluid computational domain is the idealized abdominal aortic aneurysm given in Figure 11 (left).

The whole compliant wall has a uniform thickness of 0.17 and length of 22.95 . All units are in the CGS system. The physical parameters are given by $E=6 \times 10^{6}, \nu=0.3, \rho^{\mathrm{s}}=1.2$, $\mu=0.035$ and $\rho^{\mathrm{f}}=1$. Initially, the fluid is at rest. On the inlet boundary, we impose the flow rate waveform reported in Figure 11 (right), which has been obtained form measurements at the 

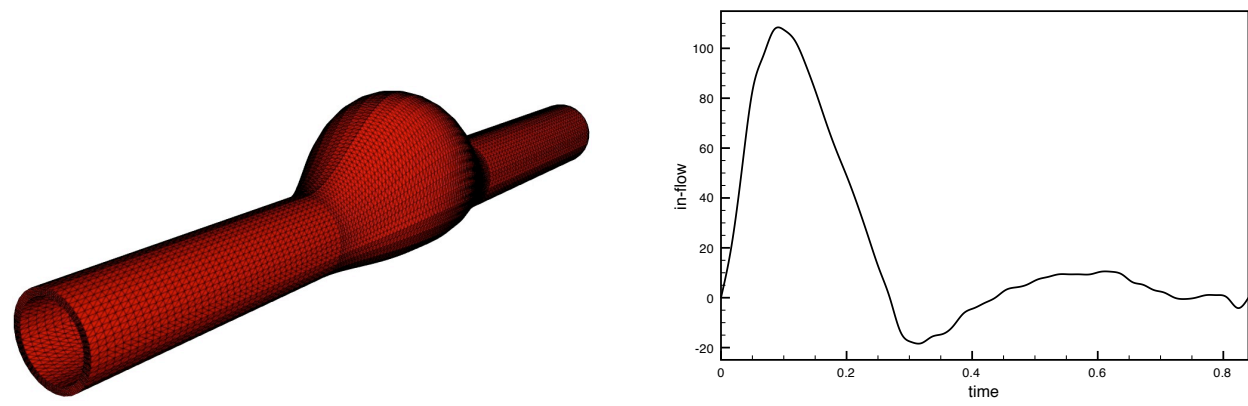

Figure 11: Aneurysm geometry (left) and in-flow rate data (right).
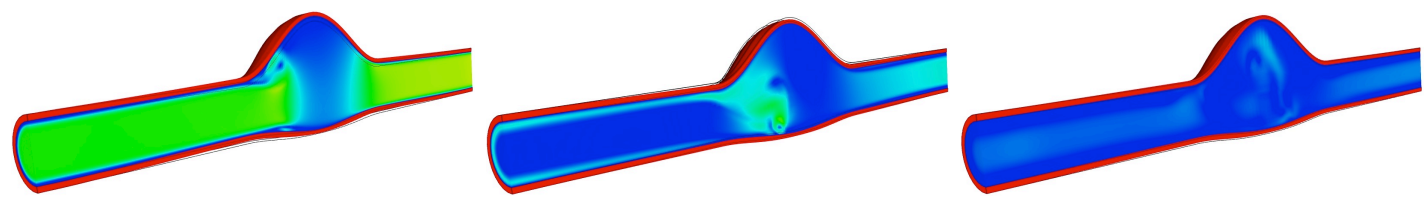

Figure 12: Snapshots of the fluid velocity and solid deformation at $t=0.2016,0.336,0.672$ (from left to right). The solid line represents the reference configuration of the solid.

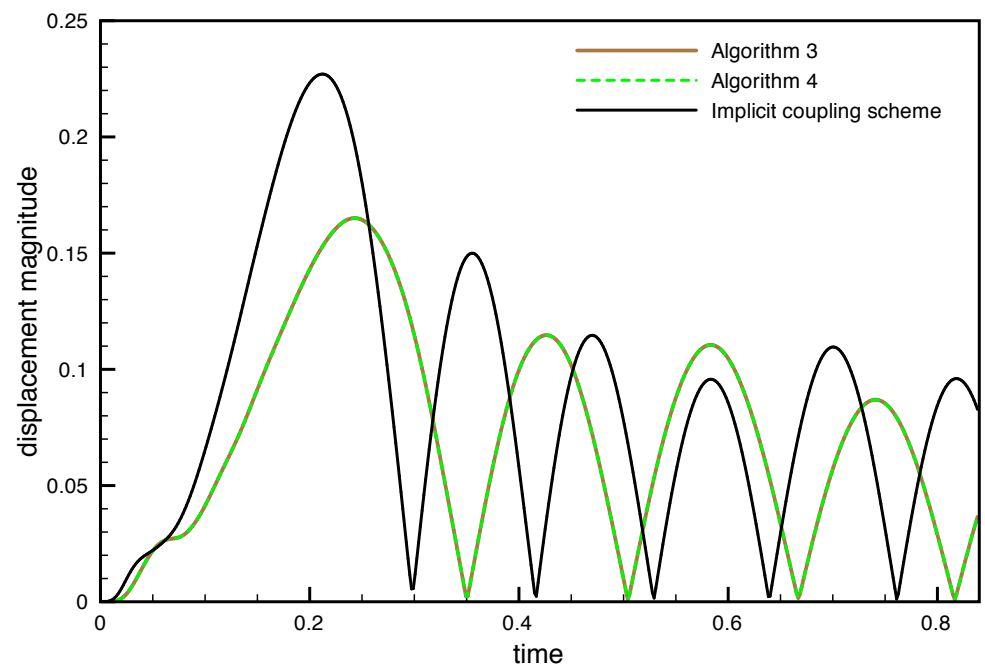

Figure 13: Comparison of the implicit and Robin-Robin explicit coupling schemes without second-order extrapolation: interface mid-point displacement magnitude vs. time.

level of the infrarenal aorta (see, e.g., [32]). A resistive-like boundary condition is prescribed on the outlet boundary, the value of the resistance being $R=300$. We have simulated 500 time-steps of size $\tau=1.68 \times 10^{-3}$, which corresponds to a complete cardiac cycle. Some snapshots of the fluid velocity and deformed fluid and solid domains are presented in Figure 12, using Algorithm 4 $\left(\gamma_{0}=1, \gamma=2500\right)$ with the second-order extrapolation and the first-order correction.

Figures 13 and 14 present the interface mid-point displacement magnitudes obtained with 


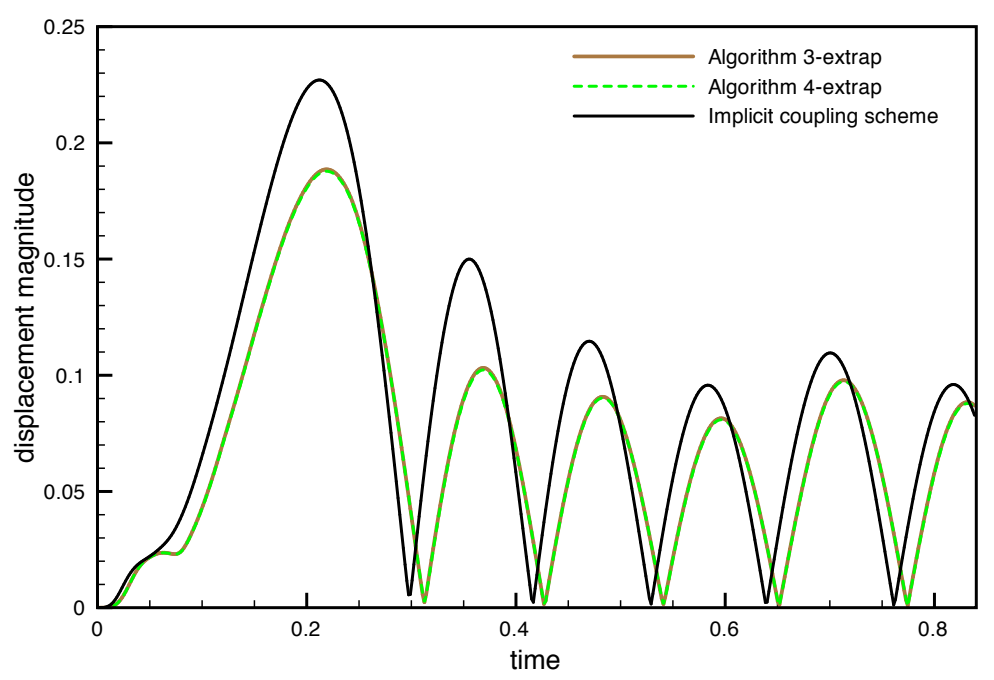

Figure 14: Comparison of the implicit and Robin-Robin explicit coupling schemes with secondorder extrapolation: interface mid-point displacement magnitude $v s$. time.

Algorithms 3 and $4^{1}$ without and with the second-order extrapolation, respectively. For comparison purposes we have also reported the solution provided by the implicit coupling scheme. Once again, the accuracy of the variants without second-order extrapolation is rather poor. The phase mismatch with the implicit coupling scheme is particularly striking. As shown in Figure 14, the Robin-Robin schemes with second-order extrapolation retrieve the overall dynamics of the solution provided by the implicit coupling scheme (in spite of the relatively large time-step $\tau$ considered). Note that the amplitude has also been improved, but it is still underestimated at the first stages of the cardiac cycle. This is due to the impact of the constant in the truncation error, which can be reduced by performing an additional correction step or by considering a shorter time-step length.

\section{Conclusion}

In this paper we have investigated several possible numerical schemes that allow for the staggered computation of fluid-structure interaction problems where the added-mass effect is strong. A salient feature of the methods suggested herein is that we observe optimal convergence order under a standard hyperbolic CFL condition for first order time discretization schemes. After comparing several numerical schemes our observations are that

- The explicit coupling can be implemented in a scheme using residual coupling terms instead of the boundary integrals characteristic for Nitsche's method.

- The main source of consistency error comes from the Nitsche penalty term, followed by the pressure stabilization term.

- Accuracy of the methods can be enhanced by using extrapolation of explicit quantities followed by one (or a few) correction $\operatorname{step}(\mathrm{s})$.

\footnotetext{
${ }^{1}$ Algorithm 2 and Algorithm 4/3 give practically the same results.
} 
- Stability and accuracy are consequences of a careful balancing of the strength of the terms imposing continuity across the interface and the pressure stabilization term relaxing the non-penetration condition. A weaker coupling may lead to a more stable scheme, in need of less stabilization. On the other hand it may result in a larger error constant and hence in reduced accuracy for a given computational mesh.

We hope that the present paper will stimulate discussion on the topic of explicit coupling methods in incompressible FSI. A field where there appears to be a number of open problems:

- High order in time: the design of an explicit coupling method of second-order remains an open problem. The naive use of higher order time discretization and second-order extrapolation in the coupling terms does not appear to be stable.

- Rigorous stability analysis: from the point of view of numerical analysis several challenging problems remain open such as the stability of Algorithms 4 and 5 .

- Accuracy of extrapolation/correction: how to prove the improved accuracy of the extrapolation method with correction remains an open problem as well as the possible dependence of algorithms on the mesh parameters and the dissipation introduced by the numerical scheme.

\section{References}

[1] M. Astorino, F. Chouly, and M. A. Fernández. Robin based semi-implicit coupling in fluidstructure interaction: Stability analysis and numerics. SIAM J. Sci. Comput., 31(6):40414065, 2009.

[2] S. Badia, F. Nobile, and C. Vergara. Fluid-structure partitioned procedures based on Robin transmission conditions. J. Comp. Phys., 227:7027-7051, 2008.

[3] Y. Bazilevs, V.M. Calo, T.J.R. Hughes, and Y. Zhang. Isogeometric fluid-structure interaction: theory, algorithms, and computations. Comput. Mech., 43(1):3-37, 2008.

[4] T. Boiveau. Penalty free Nitsche method for interface problems in computational mechanics. $\mathrm{PhD}$ thesis, University College London, in preparation.

[5] E. Burman. A penalty-free nonsymmetric Nitsche-type method for the weak imposition of boundary conditions. SIAM J. Numer. Anal., 50(4):1959-1981, 2012.

[6] E. Burman and M.A. Fernández. Stabilization of explicit coupling in fluid-structure interaction involving fluid incompressibility. Comput. Methods Appl. Mech. Engrg., 198(5-8):766$784,2009$.

[7] P. Causin, J.-F. Gerbeau, and F. Nobile. Added-mass effect in the design of partitioned algorithms for fluid-structure problems. Comput. Methods Appl. Mech. Engrg., 194(4244):4506-4527, 2005.

[8] P. Crosetto, S. Deparis, G. Fourestey, and A. Quarteroni. Parallel algorithms for fluidstructure interaction problems in haemodynamics. SIAM J. Sci. Comput., 33(4):1598-1622, 2011.

[9] S. Deparis, M. Discacciati, G. Fourestey, and A. Quarteroni. Fluid-structure algorithms based on Steklov-Poincaré operators. Comput. Methods Appl. Mech. Engrg., 195(4143):5797-5812, 2006. 
[10] W. Dettmer and D. Perić. A computational framework for fluid-rigid body interaction: finite element formulation and applications. Comput. Methods Appl. Mech. Engrg., 195(1316):1633-1666, 2006.

[11] C. Farhat, A. Rallu, K. Wang, and T. Belytschko. Robust and provably secondorder explicit-explicit and implicit-explicit staggered time-integrators for highly non-linear compressible fluid-structure interaction problems. Internat. J. Numer. Methods Engrg., 84(1):73-107, 2010.

[12] C. Farhat, K. van der Zee, and Ph. Geuzaine. Provably second-order time-accurate looselycoupled solution algorithms for transient nonlinear aeroelasticity. Comput. Methods Appl. Mech. Engrg., 195(17-18):1973-2001, 2006.

[13] M.A. Fernández. Coupling schemes for incompressible fluid-structure interaction: implicit, semi-implicit and explicit. Se $M A$ J., (55):59-108, 2011.

[14] M.A. Fernández. Incremental displacement-correction schemes for incompressible fluidstructure interaction: stability and convergence analysis. Numer. Math., 123(1):21-65, 2013.

[15] M.A. Fernández, J.F. Gerbeau, and C. Grandmont. A projection semi-implicit scheme for the coupling of an elastic structure with an incompressible fluid. Int. J. Num. Meth. Engrg., 69(4):794-821, 2007.

[16] M.A. Fernández and M. Moubachir. A Newton method using exact Jacobians for solving fluid-structure coupling. Comp. \& Struct., 83:127-142, 2005.

[17] M.A. Fernández and J. Mullaert. Displacement-velocity correction schemes for incompressible fluid-structure interaction. C. R. Math. Acad. Sci. Paris, 349(17-18):1011-1015, 2011.

[18] M.A. Fernández, J. Mullaert, and M. Vidrascu. Explicit Robin-Neumann schemes for the coupling of incompressible fluids with thin-walled structures. Comput. Methods Appl. Mech. Engrg., 2013. To appear. Preprint available at http://hal.inria.fr/hal-00784903.

[19] L. Formaggia, J.-F. Gerbeau, F. Nobile, and A. Quarteroni. On the coupling of 3D and 1D Navier-Stokes equations for flow problems in compliant vessels. Comp. Meth. Appl. Mech. Engrg., 191(6-7):561-582, 2001.

[20] L. Formaggia, A. Quarteroni, and A. Veneziani, editors. Cardiovascular Mathematics. Modeling and simulation of the circulatory system, volume 1 of Modeling, Simulation and Applications. Springer, 2009.

[21] C. Förster, W.A. Wall, and E. Ramm. Artificial added mass instabilities in sequential staggered coupling of nonlinear structures and incompressible viscous flows. Comput. Methods Appl. Mech. Engrg., 196(7):1278-1293, 2007.

[22] M.W. Gee, U. Küttler, and W. Wall. Truly monolithic algebraic multigrid for fluid-structure interaction. Int. J. Numer. Meth. Engng., 85(8):987-1016, 2011.

[23] J.-F. Gerbeau and M. Vidrascu. A quasi-Newton algorithm based on a reduced model for fluid-structure interactions problems in blood flows. Math. Model. Num. Anal., 37(4):631648, 2003.

[24] G. Guidoboni, R. Glowinski, N. Cavallini, and S. Canic. Stable loosely-coupled-type algorithm for fluid-structure interaction in blood flow. J. Comp. Phys., 228(18):6916-6937, 2009 . 
[25] M. Heil. An efficient solver for the fully coupled solution of large-displacement fluid-structure interaction problems. Comput. Methods Appl. Mech. Engrg., 193(1-2):1-23, 2004.

[26] G. Hou, J. Wang, and A. Layton. Numerical methods for fluid-structure interaction-a review. Commun. Comput. Phys., 12(2):337-377, 2012.

[27] U. Küttler and W.A. Wall. Fixed-point fluid-structure interaction solvers with dynamic relaxation. Comp. Mech., 43(1):61-72, 2008.

[28] P. Le Tallec and J. Mouro. Fluid structure interaction with large structural displacements. Comput. Meth. Appl. Mech. Engrg., 190:3039-3067, 2001.

[29] R.L. Muddle, M. Mihajlović, and M. Heil. An efficient preconditioner for monolithicallycoupled large-displacement fluid-structure interaction problems with pseudo-solid mesh updates. J. Comput. Phys., 231(21):7315-7334, 2012.

[30] F. Nobile and C. Vergara. An effective fluid-structure interaction formulation for vascular dynamics by generalized Robin conditions. SIAM J. Sci. Comput., 30(2):731-763, 2008.

[31] F. Nobile and C. Vergara. Partitioned Algorithms for Fluid-Structure Interaction Problems in Haemodynamics. Milan J. Math., 80(2):443-467, 2012.

[32] S. Oyre, E.M. Pedersen, S. Ringgaard, P. Boesiger, and W.P. Paaske. In vivo wall shear stress measured by magnetic resonance velocity mapping in the normal human abdominal aorta. Eur. J. Vasc. Endovasc. Surg., 13:263-271, 1997.

[33] K.C. Park, C.A. Felippa, and J.A. Deruntz. Stabilization of staggered solution procedures for fluid-structure interaction analysis. In Belytschko. T. and T.L. Geers, editors, Computational Methods for Fluid-Structure Interaction Problems, volume 26, pages 95-124. American Society of Mechanical Engineers, 1977.

[34] S. Piperno. Explicit/implicit fluid/structure staggered procedures with a structural predictor and fluid subcycling for $2 \mathrm{D}$ inviscid aeroelastic simulations. Internat. J. Numer. Methods Fluids, 25(10):1207-1226, 1997.

[35] O. Pironneau, F. Hecht, A. Le Hyaric, and J. Morice. Freefem++, www.freefem.org/ff++.

[36] A. Quaini and A. Quarteroni. A semi-implicit approach for fluid-structure interaction based on an algebraic fractional step method. Math. Models Methods Appl. Sci., 17(6):957-983, 2007.

[37] A.-V. Salsac, S.R. Sparks, J.M. Chomaz, and J.C. Lasheras. Evolution of the wall shear stresses during the progressive enlargement of symmetric abdominal aortic aneurysms. $J$. Fluid Mech., 550:19-51, 2006.

[38] C.M. Scotti, A.D. Shkolnik, S.C. Muluk, and E.A. Finol. Fluid-structure interaction in abdominal aortic aneurysms: effects of asymmetry and wall thickness. BioMedical Engineering OnLine, 4(1):1-22, 2005. 


\section{Contents}

1 Introduction $\quad 3$

2 A linear model problem $\quad 4$

3 Time-discretization: explicit coupling procedures $\quad \mathbf{5}$

3.1 The Nitsche based explicit coupling procedure . . . . . . . . . . . . . . . . 5

3.2 Stabilized Robin-Robin explicit coupling . . . . . . . . . . . . . . . . . . . 7

3.2 .1 Stability analysis. . . . . . . . . . . . . . . . . . . . . . 9

3.3 Genuine Robin-Robin explicit coupling . . . . . . . . . . . . . . . . . 12

4 Enhancing accuracy $\quad 12$

4.1 Second-order extrapolation with first-order correction . . . . . . . . . . . . 13

4.2 Non-symmetric penalty-free formulation . . . . . . . . . . . . . . . . . 13

4.3 Penalty free explicit coupling scheme using the non-symmetric Nitsche method without pressure stabilization . . . . . . . . . . . . . . . . . 14

5 Numerical investigations $\quad \mathbf{1 5}$

5.1 Convergence study in a two-dimensional benchmark . . . . . . . . . . . . . 15

5.2 Three-dimensional test cases . . . . . . . . . . . . . . . . . . . 19

5.2.1 Pressure wave propagation in a straight cylindrical vessel. . . . . . . . . 19

5.2.2 A physiological test-case. . . . . . . . . . . . . . . 20

6 Conclusion $\quad 22$ 


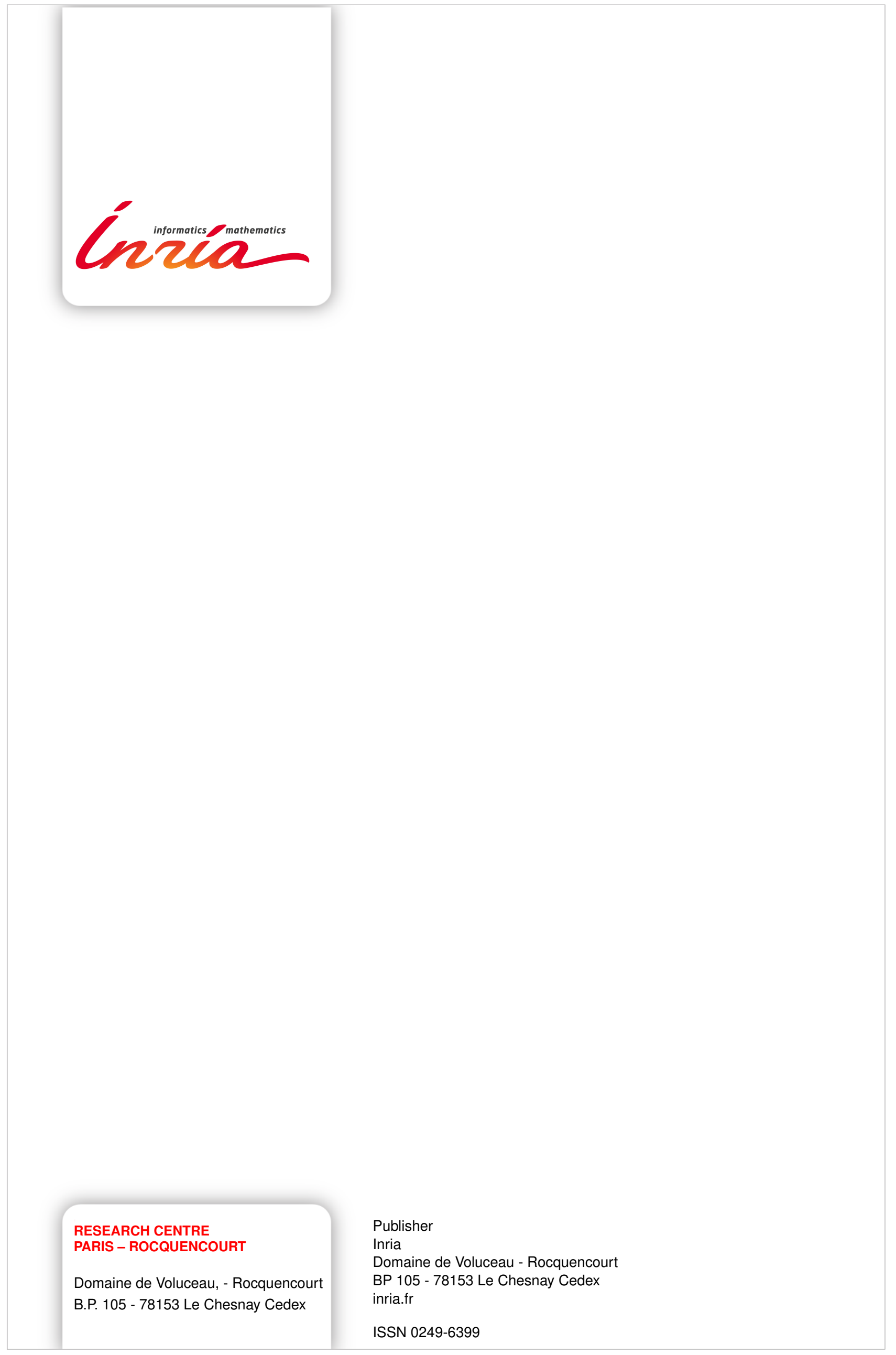

